organic communications

\title{
Evaluation of new 2-hydroxy-N-(4-oxo-2-substituted phenyl-1,3- thiazolidin-3-yl)-2-phenylacetamide derivatives as potential antimycobacterial agents
}

\section{Özlen Güzel-Akdemir ${ }^{(1, *}$, Kübra Demir-Yazıcı ${ }^{\oplus}$, Muhammed Trawally ${ }^{\oplus}$, Serap İpek Dingiş-Birgül ${ }^{2,3}$ and Atilla Akdemir $\oplus^{2}$}

\author{
${ }^{1}$ Department of Pharmaceutical Chemistry, Faculty of Pharmacy, Istanbul University, Beyazlt, 34116, \\ Istanbul, Türkiye \\ ${ }^{2}$ Computer-Aided Drug Discovery Laboratory, Department of Pharmacology, Faculty of Pharmacy, \\ Bezmialem Vakif University, Fatih, 34093, Istanbul, Türkiye \\ ${ }^{3}$ Department of Pharmaceutical Chemistry, Faculty of Pharmacy, Marmara University, Basibuyuk,
} 34854, Istanbul, Türkiye

(Received May 13, 2020; Revised May 22, 2020; Accepted May 27,2020)

\begin{abstract}
A small collection of 2-hydroxy- $N$-(5-methyl/nonsubstituted 4-oxo-2-substituted phenyl-1,3-thiazolidin-3yl)-2-phenylacetamides (3-16) was synthesized from the cyclocondensation of 2-hydroxy-2-phenyl- $N$ '[(substitutedphenyl)methylene]acetohydrazides (2) and mercaptoethanoic acid or 2-mercaptopropanoic acid, characterized with spectral and elemental analysis. In order to explore their antimycobacterial potential, newly synthesized fourteen compounds were screened for their inhibitory activity against Mycobacterium tuberculosis strain $\mathrm{H} 37 \mathrm{Rv}$ at $6.25 \mu \mathrm{g} / \mathrm{mL}$ with in-vitro primary tests. Compound 7 was found to provide the highest inhibition (98\%) $M$. tuberculosis strain H37Rv, while most of the new derivatives showed different inhibition ratios. For the search of the putative targets which are considered as related to the antimycobacterial activity of these molecules, docking studies were performed. With molecular dynamic simulations, further possible interactions between ligands and the active site of the selected enzymes were investigated. Eventually, molecular modelling studies indicated that at least part of the mechanism of action of these compounds may be mediated by inhibition of MtInhA.
\end{abstract}

Keywords: Mycobacterium tuberculosis; 2-hydroxy-2-phenylacetohydrazide; mercaptoethanoic acid; 2mercaptopropanoic acid; 4-oxo-1,3-thiazolidines; molecular modelling. @2020 ACG Publication. All right reserved.

\section{Introduction}

Tuberculosis (TB) is an infectious disease caused by the pathogenic bacterium Mycobacterium tuberculosis (MT), which spreads through the aerosol droplets contaminated by MT and affects the lungs. TB has a number of symptoms like prolonged cough, chest pain, weakness, weight loss and fever. It is primarily a pulmonary infection but can affect extrapulmonary sites including bones, the central nervous system, and many other organ systems. ${ }^{1,2}$ According to the World Health Organization (WHO) data, TB

* Corresponding author: E-Mail: oguzel@ istanbul.edu.tr, Phone: +90 2124555700 Ext. 13440 
is one of the top 10 causes of death worldwide and a total of 1.5 million people died from TB in 2018 . Especially the 30 high TB burden countries like India, China, Indonesia and others accounted for $87 \%$ of new TB cases. ${ }^{3,4}$ In addition, studies about the predicted impact of the COVID-19 pandemic on global TB cases and deaths have suggested that TB case detection has decreased by an average of $25 \%$ over 3 months (as compared to the level of detection before the pandemic). This would lead to an additional 190 $000(56000-406000) \mathrm{TB}$ deaths (a 13\% increase), bringing the total to $1.66(1.3-2.1)$ million TB deaths in $2020 .^{5}$

Today, TB is treated by the standard 6 month course of 4 antibiotics. In the standard therapy, a cocktail of first-line drugs isoniazid (INH), rifampin (RIF), pyrazinamide (PZA) and ethambutol (EMB) are given for six months. If the treatment does not reach the success because of bacterial drug resistance, or intolerance to one or more drugs, second-line drugs are used, such as para-amino-salicylate (PAS), kanamycin, fluoroquinolones, capreomycin, ethionamide and cycloserine, which are usually either less effective or more toxic with serious side effects. ${ }^{6}$ Although it is curable, the drug resistance in $M$. Tuberculosis is the major problem as a result of non-adherence to the treatment and chromosomal mutations in Tubercle bacilli that have made them resistant to every drug used to treat tuberculosis. ${ }^{7,8}$

The Mycobacterium tuberculosis enoyl-acyl carrier protein reductase (MtInhA) is an essential enzyme of the type II fatty acid synthase (FAS-II) system, which is the target for well-known first-line antitubercular drug isoniazid (INH) and of the second-line drug ethionamide (ETH). ${ }^{9}$ Mycolic acid, the major component of mycobacterial cell walls, is synthesized through the function of type I (FAS-I) and type II (FAS-II) synthase systems, which utilizes long-chain fatty acids as the raw material. FAS-I system provides the synthesis of fatty acids 16-24 carbons in length and then FAS-II system extends the fatty acid chain, and further to form the mycolic acids. ${ }^{10}$ MtInhA catalyzes the NADH-dependent reduction of the double bond of 2-trans-enoyl-[acyl-carrier protein], which is an important step at FAS-II pathway to extend fatty acid chain. ${ }^{11}$ As a result of inhibition of mycolic acid synthesis, the mycobacterial cell membrane is not able to protect its integrity and survival of the pathogenic bacteria is no more possible. Therefore, inhibition of MtInhA enzyme presents a promising target for new antimycobacterial candidates.

1,3-thiazolidin-4-one derivatives or 4-thiazolidinones namely, exhibit a wide variety of biological properties such as analgesic, ${ }^{12}$ antibacterial, ${ }^{13}$ antiparasitic, ${ }^{14}$ antioxidant, ${ }^{15}$ anticonvulsant, ${ }^{16}$ anti-HIV,${ }^{17}$ antifungal,${ }^{18}$ anticancer ${ }^{19}$ and in particularly antimycobacterial ${ }^{20,21}$ activities. In our previous study, some 4-thiazolidinone containing compounds were synthesized and evaluated for their human carbonic anhydrase IX (hCA IX) inhibitory activity as potential anticancer agents. ${ }^{22}$ Again in our very recent work, a different series of 1,3-thiazolidin-4-one derivatives were investigated as inhibitors of fungal carbonic anhydrases of Candida spp. and also tested against some Gram-positive and Gram-negative bacteria. ${ }^{23}$

In view of the fact that 1,3-thiazolidine ring possesses antimycobacterial activity and as part of our previous studies, in this project, fourteen new 2-hydroxy- $N$-(5-methyl/nonsubstituted 4-oxo-2substituted phenyl-1,3-thiazolidin-3-yl)-2-phenylacetamide derivatives were synthesized and tested for their antimycobacterial activity against Mycobacterium tuberculosis strain $\mathrm{H} 37 \mathrm{Rv}$ at $6.25 \mu \mathrm{g} / \mathrm{mL}$ with invitro primary tests. Subsequently, molecular modelling and dynamic studies were performed to elucidate the interactions between ligands and MtInhA (4BGE), MtInhA (4TZK) and MtInhA (4BQP) active sites, the enzymes which are considered as potentially related to the antimycobacterial activity.

\section{Experimental}

\subsection{Chemical Material and Apparatus}

DL 2-hydroxy-2-phenylacetic acid methyl ester, mercaptoacetic acid, 2-mercaptopropionic acid, and aromatic aldehydes were purchased from commercial sources. 2-Hydroxy-2-phenylacetohydrazide (1) was synthesized as reported in the literature. ${ }^{24}$ Uncorrected melting points were recorded using Büchi 530 apparatus and reported in degree centigrade. Leco 932 elemental analyzer was used to conduct elemental analyses. Infrared (IR) spectra were recorded as thin $\mathrm{KBr}$ pellets on Perkin Elmer Model 1600 FT-IR spectrophotometer. The values of the frequencies were expressed in $\mathrm{cm}^{-1} .{ }^{1} \mathrm{H}-\mathrm{NMR}$ and ${ }^{13} \mathrm{C}-\mathrm{NMR}$ (APT) measurements were assessed in DMSO- $d_{6}$ with Varian Inova 500 , Bruker DPX $400 \mathrm{MHz}$ instruments. Mass spectra (LC-MS-APCI) were performed in a negative ionization mode on a Finnigan LCQ Mass Spectrometer. 


\subsection{Chemistry}

\subsubsection{Synthesis of 2-Hydroxy-2-phenyl- $N^{\prime}-[($ substituted phenyl)methylene]acetohydrazides (2)}

A solution of $\mathbf{1}(0.006 \mathrm{~mol})$ in absolute $\mathrm{EtOH}(30 \mathrm{~mL})$ was heated with an appropriate aromatic aldehyde $(0.0066 \mathrm{~mol})$ under reflux for $4 \mathrm{~h}$ to give a precipitate which was filtered and purified by either washing it with hot EtOH or recrystallizing it from $\mathrm{EtOH}$ to yield the desired product.

2.2.2. General Method for the Synthesis of 2-Hydroxy-N-(4-oxo-2-substitutedphenyl-thiazolidin-3-yl)-2phenylacetamides (3-8) and 2-Hydroxy-N-(5-methyl-4-oxo-2-substitutedphenyl-thiazolidin-3-yl)-2phenylacetamides (9-16)

Using a Dean-Stark water separator, a mixture of $2(0.005 \mathrm{~mol})$ and 2-mercaptopropanoic acid or mercaptoethanoic acid $(0.02 \mathrm{~mol})$ was refluxed in dry benzene $(30 \mathrm{~mL})$ for $6 \mathrm{~h}$. The residue obtained after eliminating the excess of the solvent under reduced pressure was triturated with saturated $\mathrm{NaHCO}_{3}$ to achieve a complete evolution of $\mathrm{CO}_{2}$ gas. The resulting crude product was refrigerated overnight, was washed with $\mathrm{H}_{2} \mathrm{O}$, dried and recrystallized from $\mathrm{H}_{2} \mathrm{O}$ or EtOH to give the aimed compounds.

N-[2-(4-Bromophenyl)-4-oxo-1,3-thiazolidin-3-yl]-2-hydroxy-2-phenylacetamide (3): Yield 7\%; m.p. $179{ }^{\circ} \mathrm{C}$. IR $\left(\mathrm{v}, \mathrm{cm}^{-1}\right): 3254(\mathrm{NH}), 1722,1684(\mathrm{C}=\mathrm{O}) ;{ }^{1} \mathrm{H}-\mathrm{NMR}(500 \mathrm{MHz}, \delta, \mathrm{ppm}): 3.72(1 \mathrm{H}, \mathrm{dd}, J=15.8$, $3.1 \mathrm{~Hz}$, thiaz. $\left.\mathrm{C}_{5}-\mathrm{H}\right), 3.84\left(1 \mathrm{H}, \mathrm{dd}, J=15.8,1.8 \mathrm{~Hz}\right.$, thiaz. $\left.\mathrm{C}_{5}-\mathrm{H}\right), 4.94(1 \mathrm{H}, \mathrm{d}, J=4.9 \mathrm{~Hz}, \mathrm{CHOH}), 5.76(1 \mathrm{H}$, s, thiaz. $\left.\left.\mathrm{C}_{2}-\mathrm{H}\right), 6.21,6.25(1 \mathrm{H}, 2 \mathrm{~d}, J=5.1 \mathrm{~Hz}, \mathrm{OH}), 7.13-7.26(5 \mathrm{H}, \mathrm{m}, \mathrm{Ar}-\mathrm{H}), 7.352 \mathrm{H}, \mathrm{d}, J=8.8 \mathrm{~Hz}, \mathrm{Ar}-\mathrm{H}\right)$ 7.48, $7.55(1 \mathrm{H}, 2 \mathrm{~d}, J=8.3 \mathrm{~Hz}, \mathrm{Ar}-\mathrm{H}), 7.55(1 \mathrm{H}, \mathrm{d}, J=8.8 \mathrm{~Hz}, \mathrm{Ar}-\mathrm{H}), 10.28,10.33(1 \mathrm{H}, 2 \mathrm{~s}, \mathrm{CONH}) ;{ }^{13} \mathrm{C}-$ NMR [APT (decoupled), $125.6 \mathrm{MHz}, \delta, \mathrm{ppm}$ ]: 29.9 (thiaz. $\mathrm{C}_{5}$ ), 61.5 (thiaz. $\mathrm{C}_{2}$ ), $73.5(\mathrm{CH}-\mathrm{OH}), 127.4$, 128.2, 128.6, 130.9, 131.9 (ar. $\mathrm{CH}), 122.8(=\mathrm{C}-\mathrm{Br}), 138.0,141.0($ ar. $=\mathrm{C}$ ), 169.2 (amide $\mathrm{C}=\mathrm{O}), 171.5$ (thiaz. C=O); MS-APCI (150 eV, m/z, \%): 407 (43), 405 (M-H, 39), 332 (100), 331 (98), 273 (72), 271 (67), 199 (63), 197 (67). Anal. Calcd. for $\mathrm{C}_{17} \mathrm{H}_{15} \mathrm{BrN}_{2} \mathrm{O}_{3} \mathrm{~S}$ (407.28) : C, 50.10; H, 3.71; N, 6.88. Found: C, $50.50 ; \mathrm{H}, 3.71 ; \mathrm{N}, 6.76$.

N-[2-(3-Chlorophenyl)-4-oxo-1,3-thiazolidin-3-yl]-2-hydroxy-2-phenylacetamide (4): Yield 17\%; m.p. 195-196 ${ }^{\circ} \mathrm{C}$ IR $\left(v, \mathrm{~cm}^{-1}\right): 3333(\mathrm{NH}), 1710,1671(\mathrm{C}=\mathrm{O}) ;{ }^{1} \mathrm{H}-\mathrm{NMR}(500 \mathrm{MHz}, \delta, \mathrm{ppm}): 3.69-3.88(2 \mathrm{H}, \mathrm{d}$, $J=5.37 \mathrm{~Hz}$, thiaz. $\left.\mathrm{C}_{5}-\mathrm{H}\right), 4.95(1 \mathrm{H}, \mathrm{s}, \mathrm{CHOH}), 5.75\left(1 \mathrm{H}, \mathrm{s}\right.$, thiaz. $\left.\mathrm{C}_{2}-\mathrm{H}\right), 6.19(1 \mathrm{H}, \mathrm{s}, \mathrm{OH}), 7.16-7.22(5 \mathrm{H}$, $\mathrm{m}, \mathrm{Ar}-\mathrm{H}), 7.30-7.33(2 \mathrm{H}, \mathrm{m}, \mathrm{Ar}-\mathrm{H}), 7.36-7.38(1 \mathrm{H}, \mathrm{m}, \mathrm{Ar}-\mathrm{H}), 7.47(1 \mathrm{H}, \mathrm{s}, \mathrm{Ar}-\mathrm{H}), 10.35(1 \mathrm{H}, \mathrm{s}, \mathrm{CON} H)$. Anal. Calcd. for $\mathrm{C}_{17} \mathrm{H}_{15} \mathrm{ClN}_{2} \mathrm{O}_{3} \mathrm{~S} .1 / 2 \mathrm{H}_{2} \mathrm{O}$ (371.85): C, 54.51; H, 4.33; N, 7.53. Found: C, 55.17; H, 3.90; N, 7.64.

N-[2-(2,6-Dichlorophenyl)-4-oxo-1,3-thiazolidin-3-yl]-2-hydroxy-2-phenylacetamide (5): Yield 32\%; m.p. $184{ }^{\circ} \mathrm{C}$. IR $\left(v, \mathrm{~cm}^{-1}\right): 3301(\mathrm{NH}), 1713,1679(\mathrm{C}=\mathrm{O}) ;{ }^{1} \mathrm{H}-\mathrm{NMR}(500 \mathrm{MHz}, \delta, \mathrm{ppm}): 3.80(2 \mathrm{H}, \mathrm{dd}$, $J=15.8 \mathrm{~Hz}$, thiaz. $\left.\mathrm{C}_{5}-\mathrm{H}\right), 5.00(1 \mathrm{H}, \mathrm{s}, \mathrm{CHOH}), 6.11\left(1 \mathrm{H}, \mathrm{s}\right.$, thiaz. $\left.\mathrm{C}_{2}-\mathrm{H}\right), 6.26(1 \mathrm{H}, \mathrm{s}, \mathrm{OH}), 7.23(5 \mathrm{H}, \mathrm{m}$, Ar-H), $7.40(1 \mathrm{H}, \mathrm{d}, J=8.8 \mathrm{~Hz}, \mathrm{Ar}-\mathrm{H}), 7.58(1 \mathrm{H}, \mathrm{s}, \mathrm{Ar}-\mathrm{H}), 7.66(1 \mathrm{H}, \mathrm{d}, J=8.3 \mathrm{~Hz}, \operatorname{Ar}-\mathrm{H}), 10.47(1 \mathrm{H}, \mathrm{s}$, CONH). Anal. Calcd. for $\mathrm{C}_{17} \mathrm{H}_{14} \mathrm{Cl}_{2} \mathrm{~N}_{2} \mathrm{O}_{3} \mathrm{~S}$ (397.28): C, 51.40; H, 3.55; N, 7.05. Found: C, 51.19; H, 3.06; $\mathrm{N}, 6.89$

N-[2-(4-Fluorophenyl)-4-oxo-1,3-thiazolidin-3-yl]-2-hydroxy-2-phenylacetamide (6): Yield 34\%; m.p. $156{ }^{\circ} \mathrm{C}$. IR $\left(v, \mathrm{~cm}^{-1}\right): 3203(\mathrm{NH}), 1713,1673(\mathrm{C}=\mathrm{O}) ;{ }^{1} \mathrm{H}-\mathrm{NMR}(400 \mathrm{MHz}, \delta, \mathrm{ppm}): 3.69(1 \mathrm{H}, \mathrm{dd}, J=15.6$, $1.5 \mathrm{~Hz}$, thiaz. $\left.\mathrm{C}_{5}-\mathrm{H}\right), 3.83\left(1 \mathrm{H}, \mathrm{dd}, J=15.6,7.4 \mathrm{~Hz}\right.$, thiaz. $\left.\mathrm{C}_{5}-\mathrm{H}\right), 4.91(1 \mathrm{H}, \mathrm{t}, J=5.2 \mathrm{~Hz}, \mathrm{CHOH}), 5.75(1 \mathrm{H}$, s, thiaz. $\left.\mathrm{C}_{2}-\mathrm{H}\right), 6.15,6.20(1 \mathrm{H}, 2 \mathrm{~d}, J=4.9 \mathrm{~Hz}, \mathrm{OH}), 7.04-7.23(5 \mathrm{H}, \mathrm{m}, \mathrm{Ar}-\mathrm{H}), 7.35-7.42(4 \mathrm{H}, \mathrm{m}, \mathrm{Ar}-\mathrm{H})$, 10.20, $10.24(1 \mathrm{H}, 2 \mathrm{~s}, \mathrm{CONH}) ;{ }^{13} \mathrm{C}-\mathrm{NMR}$ [APT (decoupled), $125.6 \mathrm{MHz}, \delta, \mathrm{ppm}$ ]: 30.1 (thiaz. $\mathrm{C}_{5}$ ), 61.7 (thiaz. $\left.\mathrm{C}_{2}\right), 73.3(\mathrm{CHOH}), 116.0,127.5,128.2,128.6,130.9($ ar. $=\mathrm{CH}), 162.0,164.0(=\mathrm{C}-\mathrm{F}), 134.6,141.0$ $($ ar. $=\mathrm{C}), 169.1$ (amide $\mathrm{C}=\mathrm{O}), 171.3$ (thiaz. $\mathrm{C}=\mathrm{O})$; MS-APCI $(150 \mathrm{eV}, \mathrm{m} / z, \%): 345(\mathrm{M}-\mathrm{H}, 24), 271(94)$, 211 (38), 137 (100). Anal. Calcd. for $\mathrm{C}_{17} \mathrm{H}_{15} \mathrm{FN}_{2} \mathrm{O}_{3} \mathrm{~S}$ (346.37): C, 59.00; H, 4.36; N, 8.09. Found: C, 58.40; $\mathrm{H}, 4.33 ; \mathrm{N}, 7.75$.

N-[2-(4-Methoxyphenyl)-4-oxo-1,3-thiazolidin-3-yl]-2-hydroxy-2-phenylacetamide (7): Yield 13\%; m.p. $174{ }^{\circ} \mathrm{C}$. IR $\left(v, \mathrm{~cm}^{-1}\right): 3332(\mathrm{NH}), 1709,1673(\mathrm{C}=\mathrm{O}) ;{ }^{1} \mathrm{H}-\mathrm{NMR}(400 \mathrm{MHz}, \delta, \mathrm{ppm}): 3.70\left(3 \mathrm{H}, \mathrm{s}, \mathrm{OCH}_{3}\right)$, 
$3.71\left(1 \mathrm{H}, \mathrm{d}, J=16.1 \mathrm{~Hz}\right.$, thiaz. $\left.\mathrm{C}_{5}-\mathrm{H}\right), 3.84\left(1 \mathrm{H}, \mathrm{d}, J=15.4 \mathrm{~Hz}\right.$. thiaz. $\left.\mathrm{C}_{5}-\mathrm{H}\right), 4.96(1 \mathrm{H}, \mathrm{s}, \mathrm{CHOH}), 5.77(1 \mathrm{H}$, s, thiaz. $\left.\mathrm{C}_{2}-\mathrm{H}\right), 6.19(1 \mathrm{H}, \mathrm{d}, J=4.4 \mathrm{~Hz}, \mathrm{OH}), 6.90-6.98(3 \mathrm{H}, \mathrm{m}, \mathrm{Ar}-\mathrm{H}), 7.17-7.23(6 \mathrm{H}, \mathrm{m}, \mathrm{Ar}-\mathrm{H}), 10.31$ $(1 \mathrm{H}, \mathrm{s}, \mathrm{CONH}) ;{ }^{13} \mathrm{C}-\mathrm{NMR}$ [APT (decoupled), $\left.125.6 \mathrm{MHz}, \delta, \mathrm{ppm}\right]: 30.1$ (thiaz. $\left.\mathrm{C}_{5}\right), 55.7\left(\mathrm{OCH}_{3}\right), 62.3$ (thiaz. $\left.\mathrm{C}_{2}\right), 73.3(\mathrm{CH}-\mathrm{OH}), 113.2,115.6,127.4,128.1,128.5,130.1$ (ar. $\left.\mathrm{CH}\right), 140.2,141.1$ (ar. =C), 160.1 $\left(=C-\mathrm{OCH}_{3}\right), 169.4$ (amide $\left.\mathrm{C}=\mathrm{O}\right), 171.4$ (thiaz. $\left.\mathrm{C}=\mathrm{O}\right)$; MS-APCI $(150 \mathrm{eV}, \mathrm{m} / \mathrm{z}, \%): 357(\mathrm{M}-\mathrm{H}, 9), 283$ (46), 223 (19), 149 (100). Anal. Calcd. for $\mathrm{C}_{18} \mathrm{H}_{18} \mathrm{~N}_{2} \mathrm{O}_{4} \mathrm{~S}$ (358.41): C, 60.3; H, 5.06; N, 7.82. Found: C, $61.10 ; \mathrm{H}, 4.66 ; \mathrm{N}, 7.79$.

N-[2-(4-Benzyloxyphenyl)-4-oxo-1,3-thiazolidin-3-yl]-2-hydroxy-2-phenylacetamide (8): Yield 35\%; m.p. $162{ }^{\circ} \mathrm{C}$. IR $\left(v, \mathrm{~cm}^{-1}\right): 3360(\mathrm{OH}, \mathrm{NH}), 1724,1691(\mathrm{C}=\mathrm{O}) ;{ }^{1} \mathrm{H}-\mathrm{NMR}(400 \mathrm{MHz}, \delta, \mathrm{ppm}): 3.68(1 \mathrm{H}, \mathrm{d}$, $J=15.3 \mathrm{~Hz}$. thiaz. $\left.\mathrm{C}_{5}-\mathrm{H}\right), 3.81\left(1 \mathrm{H}, \mathrm{dd}, J=15.3,1.5 \mathrm{~Hz}\right.$. thiaz. $\left.\mathrm{C}_{5}-\mathrm{H}\right), 4.94,4.95(1 \mathrm{H}, 2 \mathrm{~s}, \mathrm{CHOH}), 5.11$, $5.14\left(2 \mathrm{H}, 2 \mathrm{~s}, \mathrm{OCH}_{2}\right), 5.73\left(1 \mathrm{H}, \mathrm{s}\right.$, thiaz. $\left.\mathrm{C}_{2}-\mathrm{H}\right), 6.40(1 \mathrm{H}$, br s, OH), 6.92-7.00 $(2 \mathrm{H}, \mathrm{m}, \mathrm{Ar}-\mathrm{H}), 7.08-7.62$ $(13 \mathrm{H}, \mathrm{m}, \mathrm{Ar}-\mathrm{H}), 10.45(1 \mathrm{H}, \mathrm{s}, \mathrm{CONH})$. Anal. Calcd. for $\mathrm{C}_{24} \mathrm{H}_{22} \mathrm{~N}_{2} \mathrm{O}_{4} \mathrm{~S}$ (434.50): C, 66.34; H, 5.10; N, 6.45. Found: C, 66.33; H, 5.05; N, 6.43.

$\mathrm{N}$-[2-(4-Bromophenyl)-5-methyl-4-oxo-1,3-thiazolidin-3-yl]-2-hydroxy-2-phenylacetamide (9): Yield 40\%; m.p. $210^{\circ} \mathrm{C}$. IR $\left(v, \mathrm{~cm}^{-1}\right): 3238(\mathrm{OH}, \mathrm{NH}), 1721,1685(\mathrm{C}=\mathrm{O}) ;{ }^{1} \mathrm{H}-\mathrm{NMR}(400 \mathrm{MHz}, \delta, \mathrm{ppm}): 1.47$ $\left(3 \mathrm{H}, \mathrm{t}, J=6.3 \mathrm{~Hz}\right.$, thiaz. $\left.\mathrm{C}_{5}-\mathrm{CH}_{3}\right), 4.01,4.11\left(1 \mathrm{H}, 2 \mathrm{q}, J=6.9 \mathrm{~Hz}\right.$, thiaz. $\left.\mathrm{C}_{5}-\mathrm{H}\right), 4.92(1 \mathrm{H}, \mathrm{d}, J=4.7 \mathrm{~Hz}$, $\mathrm{CHOH}), 5.73\left(1 \mathrm{H}, \mathrm{s}\right.$, thiaz. $\left.\mathrm{C}_{2}-\mathrm{H}\right), 6.23(1 \mathrm{H}, \mathrm{s}, \mathrm{OH}), 7.14-7.26(5 \mathrm{H}, \mathrm{m}, \mathrm{Ar}-\mathrm{H}), 7.34(2 \mathrm{H}, \mathrm{d}, J=8.8 \mathrm{~Hz}, \mathrm{Ar}-$ $\mathrm{H}), 7.54(2 \mathrm{H}, \mathrm{d}, J=8.4 \mathrm{~Hz}, \mathrm{Ar}-\mathrm{H}), 10.35(1 \mathrm{H}, \mathrm{s}, \mathrm{CONH})$. Anal. Calcd. for $\mathrm{C}_{18} \mathrm{H}_{17} \mathrm{BrN}_{2} \mathrm{O}_{3} \mathrm{~S}$ (421.31): C, $51.31 ; \mathrm{H}, 4.07 ; \mathrm{N}, 6.65$. Found: C, 51.73; H, 4.53; N, 6.66.

$N$-[2-(3-Chlorophenyl)-5-methyl-4-oxo-1,3-thiazolidin-3-yl]-2-hydroxy-2-phenylacetamide (10): Yield 35\%; m.p. $187{ }^{\circ} \mathrm{C}$. IR ( $\left.\mathrm{v}, \mathrm{cm}^{-1}\right): 3241(\mathrm{OH}, \mathrm{NH}), 1722,1682(\mathrm{C}=\mathrm{O})$; ${ }^{1} \mathrm{H}-\mathrm{NMR}(500 \mathrm{MHz}, \delta, \mathrm{ppm}): 1.49$, $1.51\left(3 \mathrm{H}, 2 \mathrm{~d}, J=6.8 \mathrm{~Hz}\right.$, thiaz. $\left.\mathrm{C}_{5}-\mathrm{CH}_{3}\right), 4.03,4.12\left(1 \mathrm{H}, 2 \mathrm{q}, J=7.0 \mathrm{~Hz}\right.$, thiaz. $\left.\mathrm{C}_{5}-\mathrm{H}\right), 4.95(1 \mathrm{H}, \mathrm{d}, J=6.3$ $\mathrm{Hz}, \mathrm{CHOH}), 5.76\left(1 \mathrm{H}, \mathrm{s}\right.$, thiaz. $\left.\mathrm{C}_{2}-\mathrm{H}\right), 6.27(1 \mathrm{H}, \mathrm{s}, \mathrm{OH}), 7.19-7.41(7 \mathrm{H}, \mathrm{m}, \mathrm{Ar}-\mathrm{H}), 7.43(1 \mathrm{H}, \mathrm{t}, J=8.7 \mathrm{~Hz}$, Ar-H), $7.50(1 \mathrm{H}, \mathrm{d}, J=7.3 \mathrm{~Hz}, \mathrm{Ar}-\mathrm{H}), 10.43(1 \mathrm{H}, \mathrm{s}, \mathrm{CONH}) ;{ }^{13} \mathrm{C}-\mathrm{NMR}$ [APT (decoupled), $125.6 \mathrm{MHz}, \delta$, ppm]: 20.2 (thiaz. $\mathrm{CH}_{3}$ ), 39.3 (thiaz. $\left.\mathrm{C}_{5}\right), 55.7\left(\mathrm{OCH}_{3}\right)$; 60.4 (thiaz. $\left.\mathrm{C}_{2}\right), 73.6(\mathrm{CH}-\mathrm{OH}), 127.3,127.5$, 127.6, 128.3, 128.6, 129.7, 130.9, 133.8 (ar. $\mathrm{CH}$ ), 140.6, 141.0 (ar. =C), 171.7 (amide $\mathrm{C}=\mathrm{O}$ ), 172.3 (thiaz. $\mathrm{C}=\mathrm{O}$ ). Anal. Calcd. for $\mathrm{C}_{18} \mathrm{H}_{17} \mathrm{ClN}_{2} \mathrm{O}_{3} \mathrm{~S}$ (376.86): C, 57.37; H, 4.55; N, 7.43. Found: C, 57.33; H, 4.28; N, 7.38.

$\mathrm{N}$-[2-(2,6-Dichlorophenyl)-5-methyl-4-oxo-1,3-thiazolidin-3-yl]-2-hydroxy-2-phenylacetamide (11): Yield 24\%; m.p. 193-194 ${ }^{\circ} \mathrm{C}$. IR $\left(v, \mathrm{~cm}^{-1}\right)$ : $3209(\mathrm{OH}, \mathrm{NH}), 1722,1647(\mathrm{C}=\mathrm{O}) ;{ }^{1} \mathrm{H}-\mathrm{NMR}(500 \mathrm{MHz}, \delta$, ppm): $1.48\left(3 \mathrm{H}, \mathrm{q}, J=6.83 \mathrm{~Hz}\right.$, thiaz. $\left.\mathrm{C}_{5}-\mathrm{CH}_{3}\right), 4.03,4.13\left(1 \mathrm{H}, 2 \mathrm{q}, J=6.83 \mathrm{~Hz}\right.$, thiaz. $\left.\mathrm{C}_{5}-\mathrm{H}\right), 5.10(1 \mathrm{H}, \mathrm{s}$, $\mathrm{CHOH}), 5.86\left(1 \mathrm{H}, \mathrm{d}, J=5.61 \mathrm{~Hz}\right.$, thiaz. $\left.\mathrm{C}_{2}-\mathrm{H}\right), 6.38(1 \mathrm{H}, \mathrm{s}, \mathrm{OH}), 7.26-7.31(1 \mathrm{H}, \mathrm{m}, \mathrm{Ar}-\mathrm{H}), 7.36(1 \mathrm{H}, \mathrm{t}$, $J=7.81 \mathrm{~Hz}, \mathrm{Ar}-\mathrm{H}), 7.41(2 \mathrm{H}, \mathrm{t}, J=7.81 \mathrm{~Hz}, \mathrm{Ar}-\mathrm{H}), 7.49(1 \mathrm{H}, \mathrm{d}, J=7.81 \mathrm{~Hz}, \mathrm{Ar}-\mathrm{H}), 7.51-7.54(3 \mathrm{H}, \mathrm{m}, \mathrm{Ar}-$ $\mathrm{H}), 11.67,11.78(1 \mathrm{H}, 2 \mathrm{~s}, \mathrm{CONH})$. Anal. Calcd. for $\mathrm{C}_{18} \mathrm{H}_{16} \mathrm{Cl}_{2} \mathrm{~N}_{2} \mathrm{O}_{3} \mathrm{~S}(411.30)$ : C, 52.56; H, 3.92; N, 6.81 . Found: C, 54.33; H, 3.21; N, 6.42 .

N-[2-(4-Fluorophenyl)-5-methyl-4-oxo-1,3-thiazolidin-3-yl]-2-hydroxy-2-phenylacetamide (12): Yield 43\%; m.p. $186{ }^{\circ} \mathrm{C}$. IR $\left(v, \mathrm{~cm}^{-1}\right): 3552,3226(\mathrm{OH}, \mathrm{NH}), 1714,1668(\mathrm{C}=\mathrm{O}) ;{ }^{1} \mathrm{H}-\mathrm{NMR}(400 \mathrm{MHz}, \delta$, ppm): 1.49, $1.50\left(3 \mathrm{H}, 2 \mathrm{~d}, J=7.0 \mathrm{~Hz}\right.$, thiaz. $\left.\mathrm{C}_{5}-\mathrm{CH}_{3}\right), 4.03,4.09\left(1 \mathrm{H}, 2 \mathrm{q}, J=6.2 \mathrm{~Hz}\right.$, thiaz. $\left.\mathrm{C}_{5}-\mathrm{H}\right), 4.93(1 \mathrm{H}, \mathrm{d}$, $J=4.3 \mathrm{~Hz}, \mathrm{CHOH}), 5.76\left(1 \mathrm{H}\right.$, s, thiaz. $\left.\mathrm{C}_{2}-\mathrm{H}\right), 6.26(1 \mathrm{H}, \mathrm{d}, J=4.5 \mathrm{~Hz}, \mathrm{OH}), 7.17-7.27(7 \mathrm{H}, \mathrm{m}, \mathrm{Ar}-\mathrm{H}), 7.42-$ $7.46(2 \mathrm{H}, \mathrm{m}, \mathrm{Ar}-\mathrm{H}), 10.36(1 \mathrm{H}, \mathrm{s}, \mathrm{CONH})$. Anal. Calcd. for $\mathrm{C}_{18} \mathrm{H}_{17} \mathrm{FN}_{2} \mathrm{O}_{3} \mathrm{~S}$ (360.40): C, 59.99; H, 4.75; N, 7.77. Found: C, 59.49; H, 4.97; N, 7.54.

N-[2-(3-Methoxyphenyl)-5-methyl-4-oxo-1,3-thiazolidin-3-yl]-2-hydroxy-2-phenylacetamide (13): Yield 18\%; m.p. $165^{\circ} \mathrm{C}$. IR ( $\left.v, \mathrm{~cm}^{-1}\right): 3565,3212(\mathrm{OH}, \mathrm{NH}), 1725,1688(\mathrm{C}=\mathrm{O}) ;{ }^{1} \mathrm{H}-\mathrm{NMR}(500 \mathrm{MHz}, \delta$, ppm): $1.48\left(3 \mathrm{H}, \mathrm{d}, J=6.83 \mathrm{~Hz}\right.$, thiaz. $\left.\mathrm{C}_{5}-\mathrm{CH}_{3}\right), 3.77\left(3 \mathrm{H}, \mathrm{s}, \mathrm{OCH}_{3}\right), 3.97,4.02\left(1 \mathrm{H}, 2 \mathrm{q}, J=6.83 \mathrm{~Hz}\right.$, thiaz. $\left.\mathrm{C}_{5}-\mathrm{H}\right)$, $4.90(1 \mathrm{H}, \mathrm{s}, \mathrm{CHOH}), 5.68\left(1 \mathrm{H}, \mathrm{s}\right.$, thiaz. $\left.\mathrm{C}_{2}-\mathrm{H}\right), 6.19(1 \mathrm{H}, \mathrm{s}, \mathrm{OH}), 6.88-6.90(2 \mathrm{H}, \mathrm{m}, \mathrm{Ar}-\mathrm{H}), 7.17-7.21(1 \mathrm{H}$, m, Ar-H), 7.22-7.26 (4H, m, Ar-H), $7.28(2 \mathrm{H}, \mathrm{d}, J=8.79 \mathrm{~Hz}, \mathrm{Ar}-\mathrm{H}), 10.24$ (1H, s, CONH). Anal. Calcd. for $\mathrm{C}_{19} \mathrm{H}_{20} \mathrm{~N}_{2} \mathrm{O}_{4} \mathrm{~S}$ (372.44): C, 61.24; H, 5.41; N, 7.52. Found: C, 60.83; H, 5.40; N, 7.38. 
N-[2-(4-Methoxyphenyl)-5-methyl-4-oxo-1,3-thiazolidin-3-yl]-2-hydroxy-2-phenylacetamide (14): Yield 16\%; m.p. $159{ }^{\circ} \mathrm{C}$. IR $\left(v, \mathrm{~cm}^{-1}\right): 3235(\mathrm{OH}, \mathrm{NH}), 1723,1682(\mathrm{C}=\mathrm{O}) ;{ }^{1} \mathrm{H}-\mathrm{NMR}(500 \mathrm{MHz}, \delta, \mathrm{ppm}): 1.50$ $\left(3 \mathrm{H}, \mathrm{d}, J=6.8 \mathrm{~Hz}\right.$, thiaz. $\left.\mathrm{C}_{5}-\mathrm{CH}_{3}\right), 3.76\left(3 \mathrm{H}, \mathrm{s}, \mathrm{OCH}_{3}\right), 4.00,4.07\left(1 \mathrm{H}, 2 \mathrm{q}, J=6.8 \mathrm{~Hz}\right.$, thiaz. $\left.\mathrm{C}_{5}-\mathrm{H}\right), 4.94$ $(1 \mathrm{H}, \mathrm{d}, J=3.4 \mathrm{~Hz}, \mathrm{CHOH}), 5.73\left(1 \mathrm{H}, \mathrm{s}\right.$, thiaz. $\left.\mathrm{C}_{2}-\mathrm{H}\right), 6.23(1 \mathrm{H}, \mathrm{s}, \mathrm{OH}), 6.93-7.20(3 \mathrm{H}, \mathrm{m}, \mathrm{Ar}-\mathrm{H}), 7.21-$ $7.25(6 \mathrm{H}, \mathrm{m}, \mathrm{Ar}-\mathrm{H}), 10.35(1 \mathrm{H}, \mathrm{s}, \mathrm{CONH})$. Anal. Calcd. for $\mathrm{C}_{19} \mathrm{H}_{20} \mathrm{~N}_{2} \mathrm{O}_{4} \mathrm{~S} . \mathrm{H}_{2} \mathrm{O}$ (390.40): C, 58.40; H, 5.63; N, 7.17. Found: C, 58.20; H, 4.44; N, 6.96.

N-[2-(3,4-Dimethoxyphenyl)-5-methyl-4-oxo-1,3-thiazolidin-3-yl]-2-hydroxy-2-phenylacetamide (15): Yield 20\%; m.p. 169-171 ${ }^{\circ} \mathrm{C}$. IR $\left(v, \mathrm{~cm}^{-1}\right)$ : $3228(\mathrm{OH}, \mathrm{NH}), 1722,1688(\mathrm{C}=\mathrm{O}) ;{ }^{1} \mathrm{H}-\mathrm{NMR}(400 \mathrm{MHz}, \delta$, ppm): $1.48\left(3 \mathrm{H}, \mathrm{d}, J=4.00 \mathrm{~Hz}\right.$, thiaz. $\left.\mathrm{C}_{5}-\mathrm{CH}_{3}\right), 3.76\left(6 \mathrm{H}, \mathrm{s}, 2 \mathrm{xOCH}_{3}\right), 3.98,4.04(1 \mathrm{H}, 2 \mathrm{q}, J=6.97 \mathrm{~Hz}$, thiaz. $\left.\mathrm{C}_{5}-\mathrm{H}\right), 4.91(1 \mathrm{H}, \mathrm{d}, \mathrm{J}=4.10 \mathrm{~Hz}, \mathrm{CHOH}), 5.70\left(1 \mathrm{H}, \mathrm{d}, J=7.26 \mathrm{~Hz}\right.$, thiaz. $\left.\mathrm{C}_{2}-\mathrm{H}\right) ; 6.23(1 \mathrm{H}$, br s., $\mathrm{OH}), 6.82-$ 6.86 $(2 \mathrm{H}, \mathrm{m}, \mathrm{Ar}-\mathrm{H}), 6.99-7.01(1 \mathrm{H}, \mathrm{m}, \mathrm{Ar}-\mathrm{H}), 7.16-7.24(5 \mathrm{H}, \mathrm{m}, \mathrm{Ar}-\mathrm{H}), 10.28(1 \mathrm{H}, \mathrm{d}, J=6.37 \mathrm{~Hz}, \mathrm{CONH})$. Anal. Cald. for $\mathrm{C}_{20} \mathrm{H}_{22} \mathrm{~N}_{2} \mathrm{O}_{5} \mathrm{~S}$ (390.40): C, 59.69; H, 5.51; N, 6.96. Found: C, 59.93; H, 5.50; N, 6.56.

$N$-[2-(4-Benzyloxyphenyl)-5-methyl-4-oxo-1,3-thiazolidin-3-yl]-2-hydroxy-2-phenylacetamide (16): Yield 49\%; m.p. $175^{\circ} \mathrm{C}$. IR ( $\left.v, \mathrm{~cm}^{-1}\right)$ : $3359(\mathrm{OH}, \mathrm{NH}), 1724,1686(\mathrm{C}=\mathrm{O}) ;{ }^{1} \mathrm{H}-\mathrm{NMR}(500 \mathrm{MHz}, \delta$, ppm): 1.46-1.49 $\left(3 \mathrm{H}, \mathrm{m}\right.$, thiaz. $\left.\mathrm{C}_{5}-\mathrm{CH}_{3}\right), 3.97,4.05\left(1 \mathrm{H}, 2 \mathrm{q}, J=6.83 \mathrm{~Hz}\right.$, thiaz. $\left.\mathrm{C}_{5}-\mathrm{H}\right), 4.89-4.94(1 \mathrm{H}, \mathrm{m}, \mathrm{CHOH})$, $5.09,5.12\left(2 \mathrm{H}, 2 \mathrm{~s}, \mathrm{OCH}_{2}\right), 5.68\left(1 \mathrm{H}, \mathrm{t}, J=6.34 \mathrm{~Hz}\right.$, thiaz. $\left.\mathrm{C}_{2}-\mathrm{H}\right), 6.13(1 \mathrm{H}, \mathrm{d}, J=5.37 \mathrm{~Hz}, \mathrm{OH}), 6.91-6.93$ $(1 \mathrm{H}, \mathrm{m}, \mathrm{Ar}-\mathrm{H}), 6.96-6.99(1 \mathrm{H}, \mathrm{m}, \mathrm{Ar}-\mathrm{H}), 7.14-7.34(8 \mathrm{H}, \mathrm{m}, \mathrm{Ar}-\mathrm{H}), 7.39(2 \mathrm{H}, \mathrm{t}, \mathrm{J}=6.84 \mathrm{~Hz}, \mathrm{Ar}-\mathrm{H}), 7.45-$ $7.47(2 \mathrm{H}, \mathrm{m}, \mathrm{Ar}-\mathrm{H}), 10.25(1 \mathrm{H}, \mathrm{s}, \mathrm{CONH})$. Anal. Calcd. for $\mathrm{C}_{25} \mathrm{H}_{24} \mathrm{~N}_{2} \mathrm{O}_{4} \mathrm{~S}$ (448.53): C, 66.94; H, 5.39; N, 6.25. Found: C, 66.98; H, 5.80; N, 6.16.

\subsection{Biological Activity}

\subsubsection{In Vitro Evaluation of Antituberculosis Activity}

Using the BACTEC 460 radiometric system, the synthesized compounds were primarily screened for their antituberculosis activity against Mycobacterium tuberculosis strain $\mathrm{H} 37 \mathrm{Rv}$ at $6.25 \mu \mathrm{g} / \mathrm{mL}$ concentration in BACTEC 12B medium. To determine the actual MIC, only compounds that showed inhibition of at least $90 \%$ were chosen for further evaluation in the CABTEC 460 at lower concentrations. The minimum inhibitory concentration (MIC) was defined as the lowest concentration of the compounds that inhibits $99 \%$ of the inoculum. ${ }^{25}$

\subsubsection{BACTEC Radiometric Method of Susceptibility Testing}

The inocula were drawn from either an isolated positive BACTEC vial for susceptibility testing with a growth index (GI) of at least 500 or from isolates of organisms suspended through a conventional medium. After vigorously mixed with a syringe, inoculum of positive BACTEC culture $(0.1 \mathrm{~mL})$ was added to each of the vials containing $6.25 \mathrm{~g} / \mathrm{mL}$ of the test compounds. For the standard vials, $2.25 \mathrm{~g} / \mathrm{mL}$ of rifampin (RMP) and a serial dilution of 1:100 of the culture was set for control. In order to provide $\mathrm{CO}_{2}$ in the headspace, BACTEC instrument was used to test each vial immediately and incubated at $37^{\circ} \mathrm{C}$ and monitored daily with BACTEC instrument. A comparison of GI in the control reading at least 30 with that of the drug vial. This was captured as a change in GI to the drug vial. We used the following formula to interpret our results:

$$
\begin{gathered}
\Delta \text { GI control }>\Delta \text { GI drug }=\text { susceptible } \\
\Delta \text { GI control }<\Delta \text { GI drug=resistant }
\end{gathered}
$$

A definite pattern of change in GI differences was established based on the additional 1 to 2 days reading of the vials when a clear susceptibility pattern (the difference of change of GI of control and the drug bottle) was not seen when the control GI was 30.

\subsection{Molecular Modelling Studies}

The MtInhA enzyme was investigated as a possible target for the fourteen thiazolidinone compounds according to the previously described protocol. ${ }^{26}$. 


\subsubsection{Preparation of Ligands Structures}

Three-dimensional structures of all ligands and all their possible stereoisomers were prepared with MOE (v2019.01.02, Chemical Computing Group Inc., Montreal, QC, Canada) in low-energy conformations. The most prevalent protonation state of the ligands at $\mathrm{pH} 7$ was calculated. Finally, the ligands were energy minimized using a steepest-descent protocol MMFF94x force field. ${ }^{27}$

\subsubsection{Preparation of $X$-ray Structures}

The three crystal structures of Mycobacterium tuberculosis enoyl-acyl carrier protein reductase (MtInhA) complexed with 1-cyclohexyl- $N$-(3,5-dichlorophenyl)-5-oxopyrrolidine-3-carboxamide (MtInhA; PDB: 4TZK; $1.62 \AA$ A), complexed with methyl-thiazole compound (MtInhA; PDB: 4BQP; 1.89 $\AA$ A) and a mutant (S94A) complexed with pyridomycin (MtInhA(S94A); PDB: 4BGE; $2.25 \AA$ ) have been obtained from the RCSB Protein Data Bank. Buffer molecules, ions, and water molecules were deleted, and all other atoms were retained. The remaining structure was protonated according to the protonate 3D protocol found in the MOE package. ${ }^{28}$ Subsequently, the obtained structures were energy minimized by using the AMBER14:EHT force field. ${ }^{29}$

\subsubsection{Docking Studies}

Docking calculations were performed using the FlexX docking tool (v2.3.2; BioSolveIT GmbH, St. Augustin, Germany) within MOE. The binding pockets were defined as all residues within $6.5 \AA$ of the reference ligands 1-cyclohexyl- $N$-(3,5-dichlorophenyl)-5-oxopyrrolidine-3-carboxamide, methylthiazole compound and pyridomycin for the crystal structures of MtInhA (PDB:4TZK, 4BQP and 4BGE respectively). All ligands were docked fifty times, and the best-scoring three poses were subjected to refinement calculations. Subsequently, the docked ligand and binding pocket residues were energyminimized and rescored using the GBVI/WSA force field. ${ }^{30}$

\subsubsection{Molecular Dynamics Simulations}

By using the NAMD software package (v2.12, Theoretical and Computational Biophysics Group, NIH Center for Macromolecular Modeling and Bioinformatics, The Beckman Institute, University of Illinois at Urbana-Champaign), molecular dynamic (MD) simulations of suggested molecules and original ligands were performed. ${ }^{31}$ The selected docked poses (ligand-enzyme complexes) were first placed into the center of a box with periodic boundary conditions which have a minimum distance of $10 \AA$ between protein and boundary. To create a solvated and neutral system, both water molecules $\left(1.005 \mathrm{~g} / \mathrm{cm}^{3}\right)$ and counter ions $(\mathrm{NaCl})$ were added. After the minimization of all structures with a steepest-descent protocol (AMBER14:EHT), the system was first heated from 0 to $300 \mathrm{~K}$ for 50 ps followed by a 50 ps equilibration simulation (position restraints on all protein and ligand heavy atoms). Finally, the system was simulated for $2 \mathrm{~ns}$ at constant temperature $(300 \mathrm{~K}$, Langevin, default values) and pressure (1 bar, Nosé-Hoover Langevin, default values), without any position restrains. The time step was set to $0.002 \mathrm{fs}$ and all bonds were constrained using the ShakeH algorithm.

\section{Results and Discussion}

\subsection{Chemistry}

The new thiazolidinone derivatives were prepared from 2-hydroxy-2-phenylacetohydrazide (1) obtained from the reaction between DL 2-hydroxy-2-phenylacetic acid methyl ester and hydrazine hydrate. Compound 1 reacted with substituted aromatic aldehydes to afford intermediate Schiff bases (2), after that cyclodehydration with mercaptoethanoic acid and 2-mercaptopropanoic acid, targeted compounds, 2-hydroxy- $N$-(4-oxo-2-substitutedphenyl-1,3-thiazolidin-3-yl)-2-phenylacetamides (3-8) and 2-hydroxy- $N$-(5-methyl-4-oxo-2-substitutedphenyl-1,3-thiazolidin-3-yl)-2-phenylacetamides (9-16) were yielded respectively (Scheme 1). 


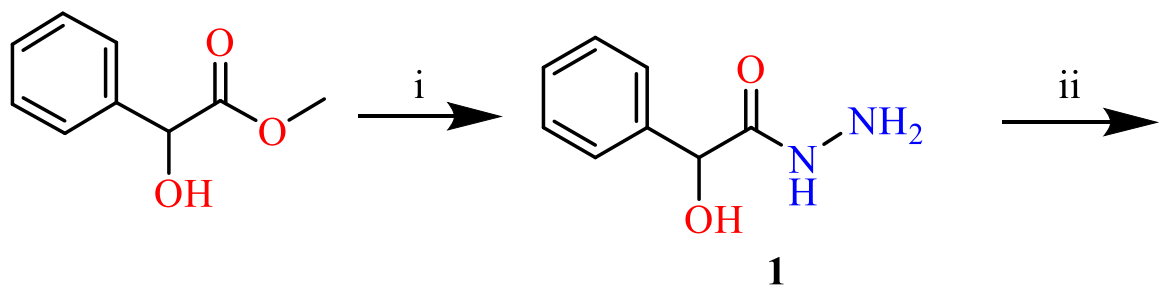

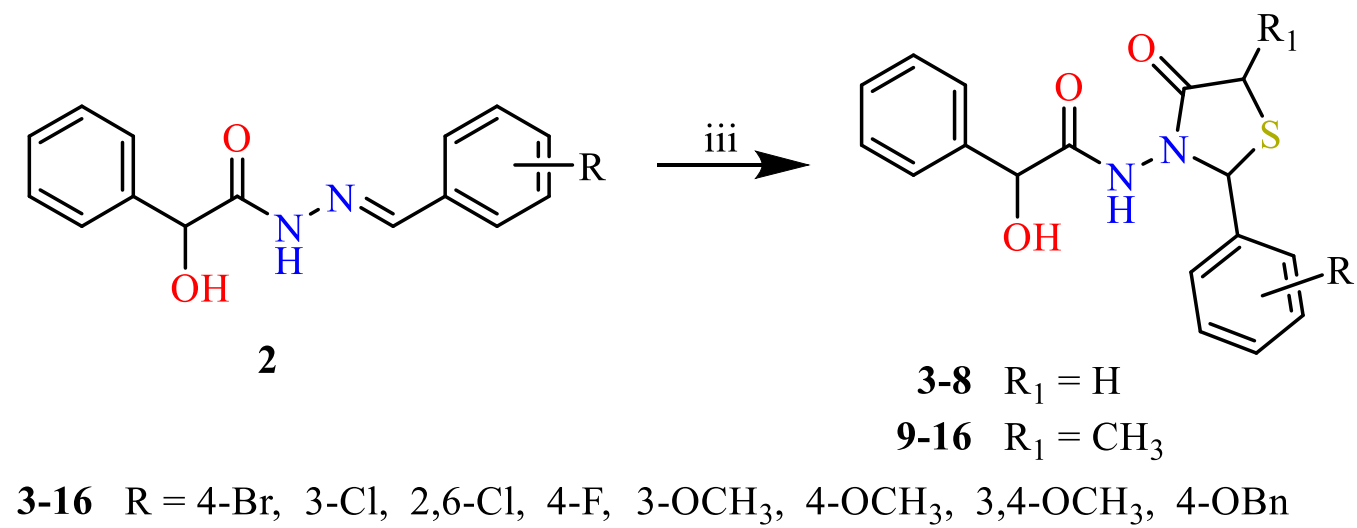

Scheme 1. Reagents: i) hydrazine hydrate, EtOH, reflux, 6h; ii) EtOH, reflux, 4h; iii) mercaptoethanoic acid / 2-mercaptopropanoic acid, dry benzene, reflux, $6 \mathrm{~h}$

The structures of 3-16 were characterized by elemental analysis and spectral data (IR, ${ }^{1} \mathrm{H}$ NMR, ${ }^{13} \mathrm{C}$ NMR and APCI Mass).

In the IR spectra of the new compounds, O-H / N-H stretching bands were observed at the 4000 $2500 \mathrm{~cm}^{-1}$ area between $3209-3565 \mathrm{~cm}^{-1}$. Strong stretching bands of new lactam $\mathrm{C}=\mathrm{O}$ groups $(1709-1725$ $\left.\mathrm{cm}^{-1}\right)$ are characteristic for 4-thiazolidinones ${ }^{32,33}$ besides $\mathrm{C}=\mathrm{O}$ amide bands $\left(1647-1691 \mathrm{~cm}^{-1}\right)$ in the IR spectra confirmed the aimed cyclization of the thiazolidinone ring. ${ }^{34}$

In the ${ }^{1} \mathrm{H}-\mathrm{NMR}$ spectrums of compounds, the methylene $\left(\mathrm{SCH}_{2}\right)$ protons as two singlets or two doublets for compounds 3-8 and methine $\left(\mathrm{SCHCH}_{3}\right)$ protons as two quartets for series 9-16 detected at about $\delta 3.68-3.84$ and $\delta 3.97-4.13 \mathrm{ppm}$ respectively, which are characteristic peaks belong to protons at 5-position of the 2,3 disubstituted 4-thiazolidinone rings. ${ }^{35}$ The $\mathrm{C}_{2}-\mathrm{H}$ protons for all compounds were appeared at about $\delta 5.68-6.11 \mathrm{ppm}$. The signals of $\mathrm{C}-\mathrm{OH}$ and $\mathrm{CONH}$ protons were signaled at about $\delta$ 6.13-7.44 and $\delta$ 10.24-11.78 ppm, respectively. Peaks associated with other protons of the molecules were observed in the expected regions. ${ }^{36}$

The ${ }^{13} \mathrm{C}$ NMR spectra of $\mathbf{3 , 6}, \mathbf{7}$ and $\mathbf{1 0}$ assigned on the basis of APT spectra and literature, showed lactam $\mathrm{C}=\mathrm{O}$ signals at $\delta 171.3-172.3 \mathrm{ppm}$ and provided further proof for thiazolidinone formation. $\mathrm{C}_{2}$ resonances of compounds $\mathbf{3 , 6}, \mathbf{7}$ and $\mathbf{1 0}$ appeared at $\delta 60.40-62.30 \mathrm{ppm}$. For compounds $\mathbf{3 ,} \mathbf{6}$ and 7, signals of $\mathrm{C}_{5}$ detected at $\delta 29.90-30.10$ and for compound 10 at $\delta 39.9 \mathrm{ppm}$. The chemical shifts of the carbons at aromatic rings observed at the aromatic area, as expected. ${ }^{37,38}$

$(\mathrm{M}-\mathrm{H})^{-}$ions with different intensities observed in the atmospheric pressure chemical ionization APCI (-) mass spectra of $\mathbf{3}, \mathbf{6}$ and $\mathbf{7}$ provided further confirmation for the formation of the expected structures. Compounds 3, $\mathbf{6}$ and $\mathbf{7}$ fragmented via two prominent pathways to afford the fragments (M$\mathrm{H})-74$ by breaking 1,2 and 3,4 bonds of the thiazolidinone ring and the fragments $(\mathrm{M}-\mathrm{H})-134$ by breaking of the C-N bond the hydrazide moiety. The proposed APCI mass fragmentation route of $\mathbf{3}, \mathbf{6}$ and $\mathbf{7}$ recorded employing the negative ionization mode is depicted in Scheme 2. 


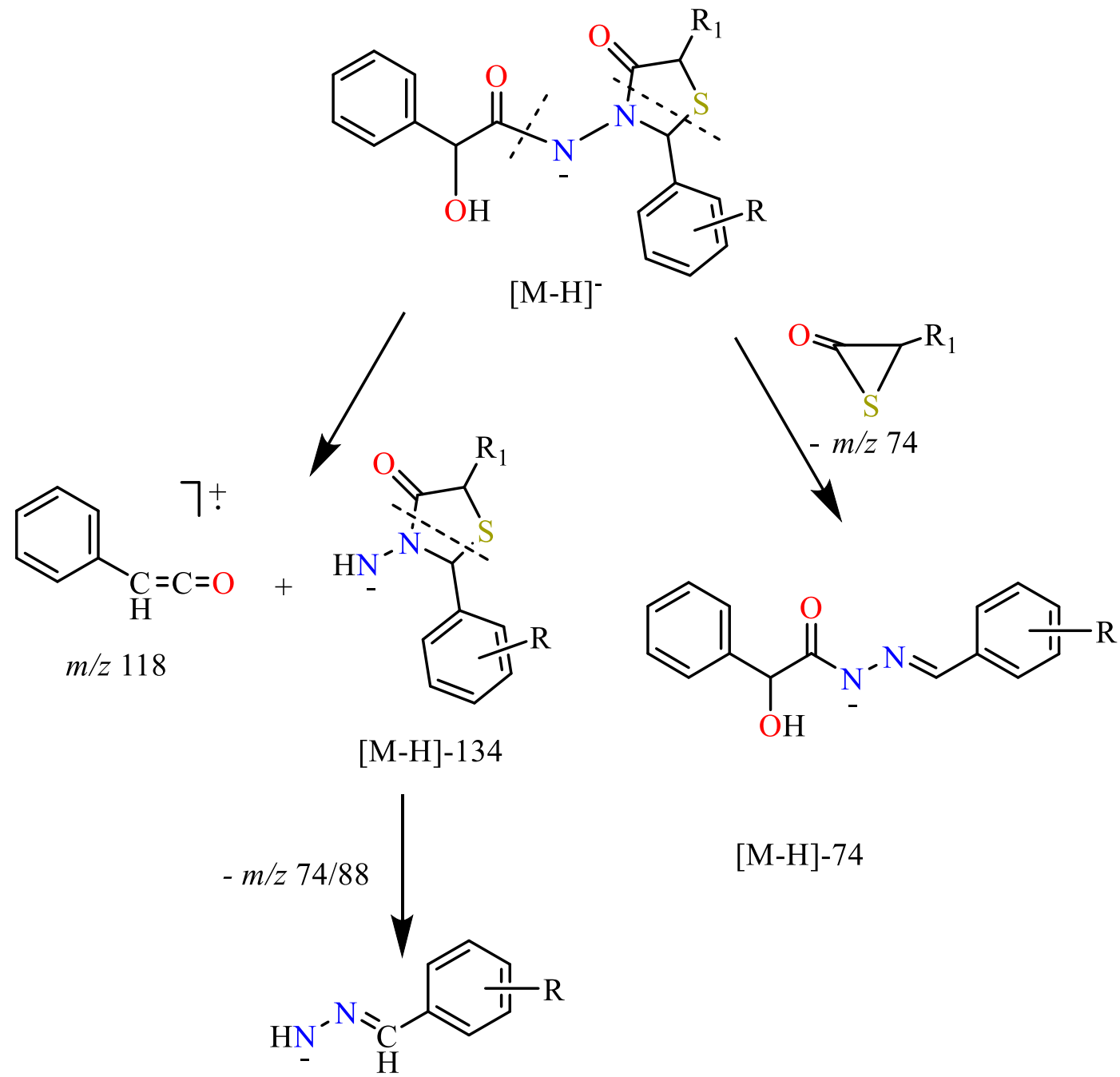

$[\mathrm{M}-\mathrm{H}]-208$

Scheme 2. Proposed mass fragmentation pattern of compounds $\mathbf{3 , 6}$ and $\mathbf{7}$

\subsection{Biological Activity}

\subsubsection{Antimycobacterial Activity}

The selected compounds were evaluated for antituberculosis activity against Mycobacterium tuberculosis strain H37Rv. Primary screening was conducted at $6.25 \mu \mathrm{g} / \mathrm{mL}$ against the microorganism in BACTEC 12B medium using the BACTEC 460 radiometric system. Rifampin was used as the standard in the tests. ${ }^{25}$ Compounds 3-16 showed varying degrees of inhibition in the primary screen (Table 1).<smiles>[R][X]c1ccccc1C1SC([R])C(=O)N1NC(=O)C(O)c1ccccc1</smiles>

Figure 1. The general chemical structures of novel compounds 3-16 
Table 1. The primary in vitro antimycobacterial activity screening results of 3-16

\begin{tabular}{ccccc}
\hline Compound & $\mathbf{R}$ & $\mathbf{R}_{\mathbf{1}}$ & $\mathbf{M I C}(\boldsymbol{\mu g} \mathbf{g} \mathbf{m L})$ & ${\text { Inhibition } \mathbf{\%}^{\mathbf{a}}}^{\mathbf{3}}$ \\
\hline $\mathbf{3}$ & $4-\mathrm{Br}$ & $\mathrm{H}$ & $>6.25$ & 6 \\
$\mathbf{4}$ & $3-\mathrm{Cl}$ & $\mathrm{H}$ & $>6.25$ & 9 \\
$\mathbf{5}$ & $2,6-\mathrm{Cl}$ & $\mathrm{H}$ & $>6.25$ & 31 \\
$\mathbf{6}$ & $4-\mathrm{F}$ & $\mathrm{H}$ & $>6.25$ & 10 \\
$\mathbf{7}$ & $4-\mathrm{OCH}_{3}$ & $\mathrm{H}$ & $>6.25$ & 98 \\
$\mathbf{8}$ & $4-\mathrm{OBn}$ & $\mathrm{H}$ & $>6.25$ & 12 \\
$\mathbf{9}$ & $4-\mathrm{Br}$ & $\mathrm{CH}_{3}$ & $>6.25$ & 6 \\
$\mathbf{1 0}$ & $3-\mathrm{Cl}$ & $\mathrm{CH}_{3}$ & $>6.25$ & 3 \\
$\mathbf{1 1}$ & $2,6-\mathrm{Cl}$ & $\mathrm{CH}_{3}$ & $>6.25$ & 0 \\
$\mathbf{1 2}$ & $4-\mathrm{F}$ & $\mathrm{CH}_{3}$ & $>6.25$ & 6 \\
$\mathbf{1 3}$ & $3-\mathrm{OCH}_{3}$ & $\mathrm{CH}_{3}$ & $>6.25$ & 11 \\
$\mathbf{1 4}$ & $4-\mathrm{OCH}_{3}$ & $\mathrm{CH}_{3}$ & $>6.25$ & 0 \\
$\mathbf{1 5}$ & $3,4-\mathrm{OCH}_{3}$ & $\mathrm{CH}_{3}$ & $>6.25$ & 22 \\
$\mathbf{1 6}$ & $4-\mathrm{OBn}$ & $\mathrm{CH}_{3}$ & $>6.25$ & 4
\end{tabular}

${ }^{\text {a }}$ MIC rifampicin $0.25 \mu \mathrm{g} / \mathrm{mL}$, 97-99\% inhibition versus $M$. tuberculosis strain H37Rv.

As reported in the table above, all the synthesized compounds were tested for their antitubercular activity against Mycobacterium tuberculosis strain H37Rv. All the compounds showed antitubercular activity except compounds $\mathbf{1 1}$ and $\mathbf{1 4}$. We assessed our SAR based on the modification of substituents of the 5th position and the substituents on the phenyl ring attached to the 2 nd position of the thiazolidinone ring.

Except for compound 9, the addition of a methyl group of position 5 of the thiazolidinone ring yielded a distinct loss of activity against the bacteria. The presence of different substituents on various positions of the phenyl ring resulted in significant changes in the inhibition percentage of the compounds. With regards to the 5-non-methyl thiazolidinone compounds (i.e., compounds 3-8), the dihalo-substitution of the phenyl ring led to an improvement in the activity when we compared compounds $\mathbf{3}, \mathbf{4}$ and $\mathbf{6}$. On the contrary, the dihalo-substitution of the phenyl ring in 5-methylthiazolidinone derivatives led to a complete loss of activity (compound 11).

Replacement of the halogens with a methoxy group on the phenyl ring significantly enhanced the activity. However, the presence of a bulkier group, a benzyloxy group, led to a deterioration in the activity when compounds 7 and 8 are compared. Comparing compounds 13, 14 and 15, the dimethoxyphenyl derivative displayed a better antimycobacterial activity than the rest.

\subsection{Molecular Modelling Studies}

To investigate MtInhA as a possible target for these compounds, we performed molecular modelling studies using the co-crystal structures of this enzyme in complex with 1-cyclohexyl- $\mathrm{N}-(3,5-$ dichlorophenyl)-5-oxopyrrolidine-3-carboxamide (4TZK), a methyl-thiazole compound (4BQP) and a mutant derivate MtInhA (S94A) complexed with pyridomycin (4BGE). These structures were chosen due to significant differences in the binding pocket. The different rotamers of Tyr 158 sidechain at the active site of the MtInhA (4TZK) and (4BQP) affect the ligand's position at the hydrophobic pocket and the possible interactions. ${ }^{39}$ With a single point mutation allele (S94A) in MtInhA is occurred the single substitution of alanine for serine 94 resulted in INH resistance, which seems to be directly related to decreases the binding affinity of the drug to $\mathrm{NAD}^{+}{ }^{40,41}$

Interesting docked poses have been obtained for the compounds in the active site of MtInhA (4TZK) and MtInhA (4BQP), but not for MtInhA (4BGE).

\subsubsection{Docking Studies and MD Simulations into the Active Site of MtInhA (4TZK)}

To explain the binding features between the MtInhA (4TZK) crystal structure and the reference ligand 1-cyclohexyl- $\mathrm{N}$-(3,5-dichlorophenyl)-5-oxopyrrolidine-3-carboxamide, docking studies and $2 \mathrm{~ns}$ MD simulation was performed (Figure 2). In crystal structures of MtInhA (4TZK), NAD ${ }^{+}$(Nicotinamide Adenine Dinucleotide) is presented as a cofactor of the enzyme. 
A

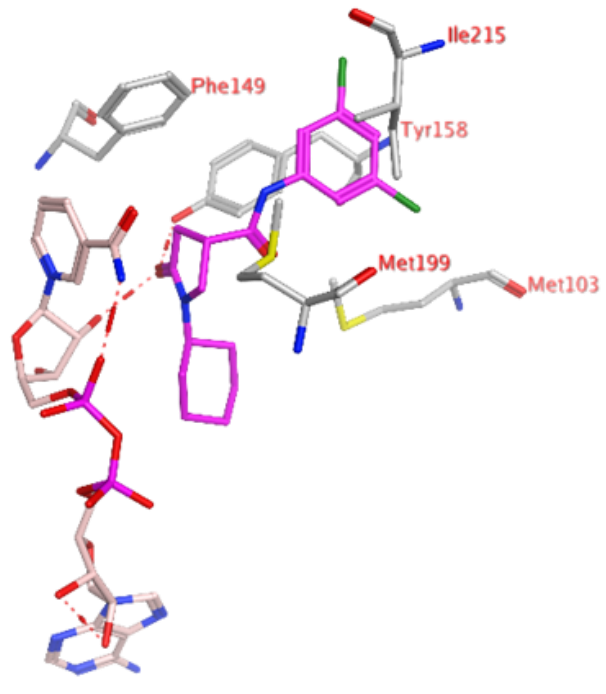

B

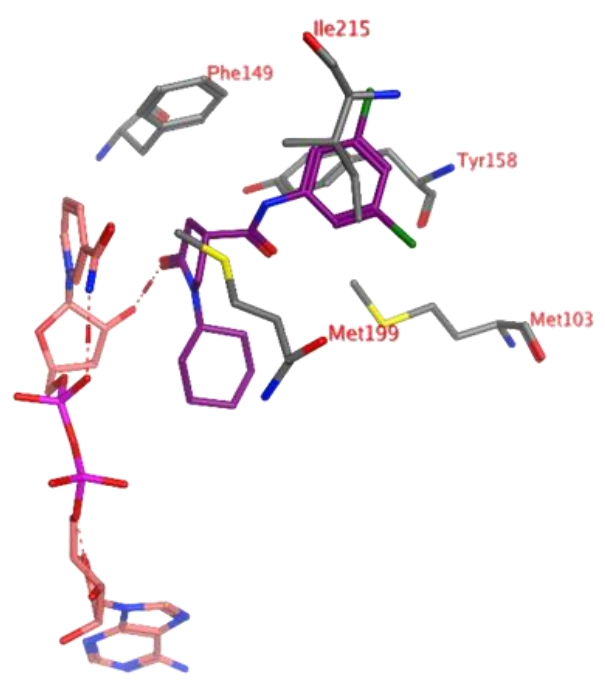

C

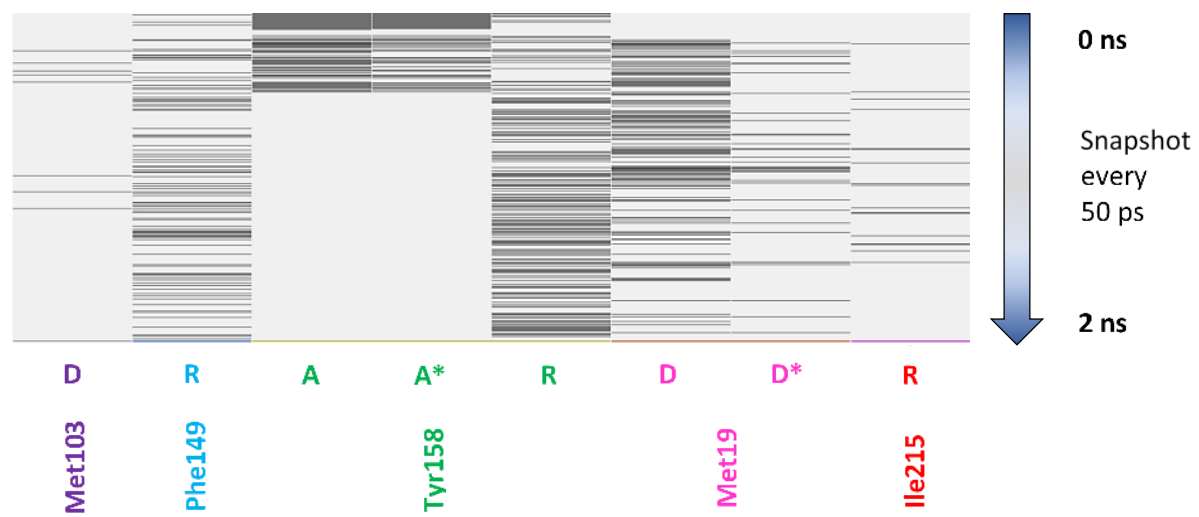

D

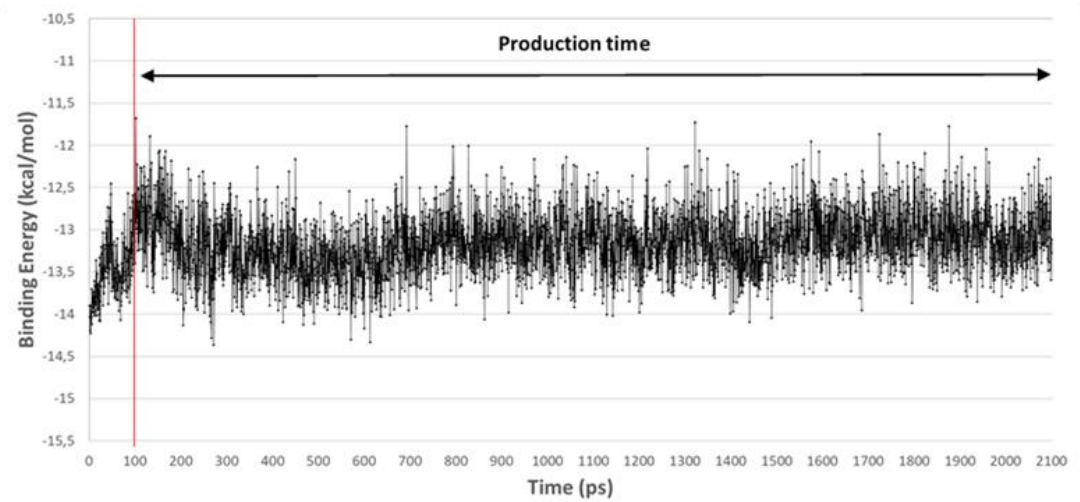

Figure 2. (A) The MtInhA (4TZK)-reference ligand (indicated purple) $(\mathrm{t}=0 \mathrm{~ns})$. $\mathrm{NAD}^{+}$is presented as a cofactor (pink). (B) The MtInhA (4TZK)-reference ligand after a 2 ns MD simulation. (C) The proteinligand interaction fingerprint (PLIF) showing the interaction of reference ligand with the MtInhA (4TZK) binding pocket residues during the simulation as barcodes (a black line indicating the presence of the interaction at snapshot). (D) The binding energy $(\mathrm{kJ} / \mathrm{mol})$ between reference ligand and MtInhA (4TZK). Hydrogen bonds are indicated in red dashed lines. " $\mathrm{D}$ " indicates side chain donor interactions, "R" indicates $\mathrm{H}-$ arene interactions and "A" indicates sidechain acceptor interactions. Two interactions of the same type per residue (for example "A" for Tyr158) indicate that multiple interactions of the specified type between ligand and residue are formed. "*" indicates the strong interactions. The arrow indicates the sequence of snapshots of every 50 ps from 0 to 2 ns. 
In docking studies, the carboxyl moiety at the thiazolidinone ring of the reference ligand form hydrogen bonds with $\mathrm{NAD}^{+}$and sidechain of Tyr158. Phenyl ring may form hydrophobic interactions or $\mathrm{H}$-arene with the sidechains of Tyr158 and Ile215 (Figure 2A).

In MD simulation, by using the protein-ligand interaction fingerprint (PLIF) tool of MOE (v2019.01.02, Chemical Computing Group Inc., Montreal, Quebec, Canada), the intermolecular interactions between the enzyme active site and reference ligand were analyzed (Figure 2C). The formed main interactions during the 2 ns simulation (represented as 40 snapshots; one snapshot per 50 ps) were with Met103 ("D": side chain donor interaction), Phe149 ("R": H-Arene interaction), Tyr158 ("A": side chain acceptor interaction and "R"), Met199 ("D") and Ile215 ("R"). The hydrogen bond between the reference ligand and the sidechain of Tyr 158 was observed in 39\% of the snapshots. Another formed hydrogen bond between the reference ligand and the sidechain of Met199 was observed in $31 \%$ of the frames, including the first $(0 \mathrm{~ns})$ and the last $(2 \mathrm{~ns})$. Interaction between reference ligand and $\mathrm{NAD}^{+}$with the distance smaller than $3.2 \AA$ accepted as moderate, ${ }^{42}$ as shown in Figure $2 \mathrm{C}$, this interaction is observed in $100 \%$ of the production run snapshots. The hydrogen bond between the lactam carbonyl group of the reference ligand and the hydroxyl group at the furan ring of the NAD+ seems to be stable during MD simulation. (Figure 2B). The calculated average binding energy between reference ligand and MtInhA (4TZK) was $-13.1 \mathrm{~kJ} / \mathrm{mol}$ with the root-mean-square deviation value (RMSD) of $0.3614 \AA$ (Figure 2D).

Compounds 3-16 have been docked into the active site of the MtInhA (4TZK) crystal structure. Interactions between MtInhA (4TZK) active site and compound 7, which has the best inhibition ratio against M.Tuberculosis strain H37Rv (98\%) is shown in Figure 3A. Hydroxyl group of the ligand forms a hydrogen bond with cofactor $\mathrm{NAD}^{+}$and another hydrogen bond between amide functionality of the ligand and the backbone of the Gly96 is observed.

During a $2 \mathrm{~ns}$ MD simulation (represented as 40 snapshots; one snapshot per $50 \mathrm{ps}$ ), the observed main interactions between ligands and active site were Gly96 ("d": backbone donor interaction, "a": backbone acceptor interaction and "R": H-Arene interaction), Phe97 and Met98 (both "a"), Met103 ("D": side chain donor interaction), Phe149 ("R") and Met199 ("D", "a" and "R") as shown in Figure 3C. The hydrogen bond between the ligand and the backbone of Gly96 was observed in $99 \%$ of the snapshots. Another formed hydrogen bond between the ligand and the backbone of Met 98 was observed in $63 \%$ of the frames, including the first ( $0 \mathrm{~ns}$ ) and the last ( $2 \mathrm{~ns})$. In addition, a hydrogen bond formed by sidechain of Met 103 is observed in 44\% of the snapshots. Although not shown in Figure 3C, the interaction between the ligand and $\mathrm{NAD}^{+}$(in a smaller distance than $3.2 \AA$ ) is observed in $74.4 \%$ of the production run snapshots. Therefore, the interaction between oxygen atom $(\mathrm{O} 1 \mathrm{~A})$ of the phosphate group at $\mathrm{NAD}^{+}$and amide nitrogen atom of the ligand may be kept. (Figure 3B). The calculated average binding energy between compound 7 and MtInhA (4TZK) was -12.9 kJ/mol (RMSD: $0.3642 \AA$ ) (Figure 3D).

\subsubsection{Docking Studies and MD Simulations into the Active Site of MtInhA(4BQP)}

With the same purpose above, docking studies and a 2 ns MD simulation was performed on the MtInhA (4BQP) crystal structure in complex with the methyl-thiazole compound as reference ligand, and $\mathrm{NAD}^{+}$(Nicotinamide Adenine Dinucleotide) is presented as a cofactor (pink) of the enzyme (Figure 4).

In docking studies, it is observed that the reference ligand interacts with $\mathrm{NAD}^{+}$and forms a hydrogen bond with the backbone of Met98. Dichloro-phenyl ring of the ligand located at a hydrophobic pocket and may interact with Tyr158 and Ile202 backbones (Figure 4A).

The main detected interactions during $2 \mathrm{~ns}$ the simulation (represented as 40 snapshots; one snapshot per $50 \mathrm{ps}$ ) were with Gln103 (“D”: sidechain donor interaction), Met103 ("R": H-Arene interaction), Ala198 ("d": backbone donor interaction) and Ile202 ("R"), analyzed with PLIF tool of MOE (Figure 4C). The hydrogen bond between reference ligand and the sidechains of Met103 and Ala198 were observed in 39\% and 30\% of the snapshots, respectively. Although not shown in Figure 4C, three different interactions between the ligand and $\mathrm{NAD}^{+}$are detected during the $\mathrm{MD}$ simulation, again in a smaller distance than $3.2 \AA$. 
$\mathbf{A}$
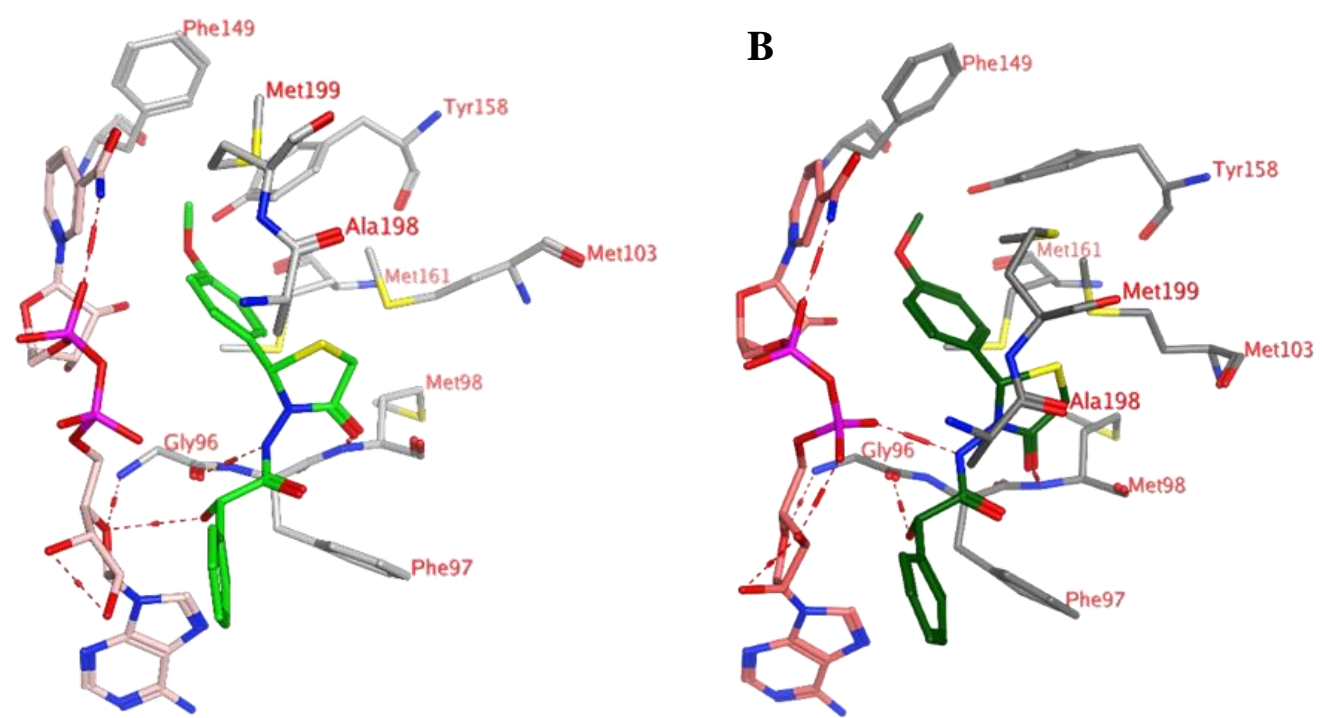

C

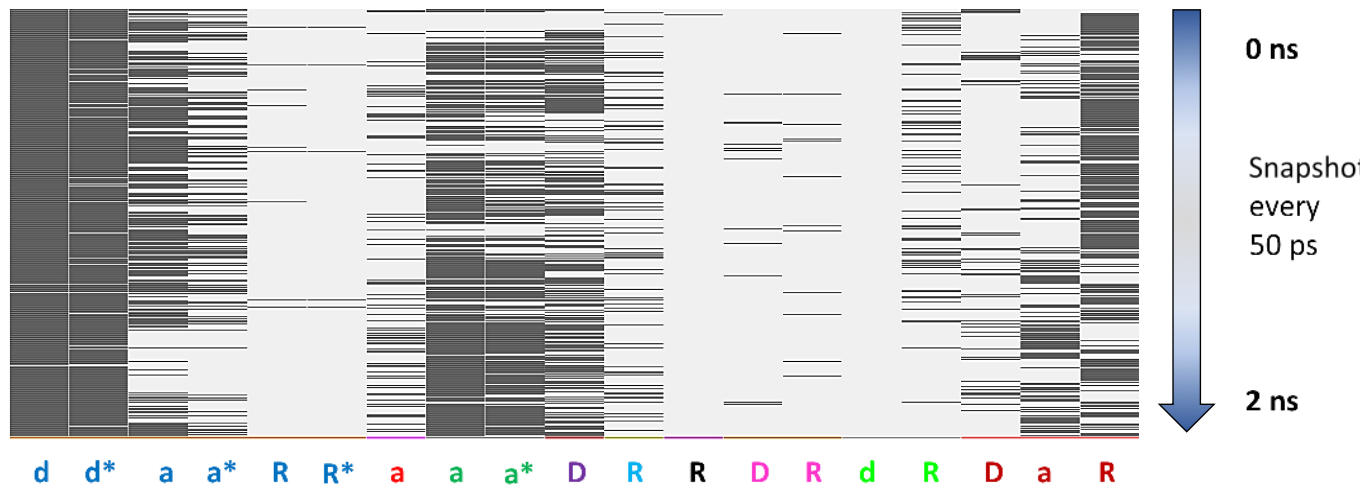

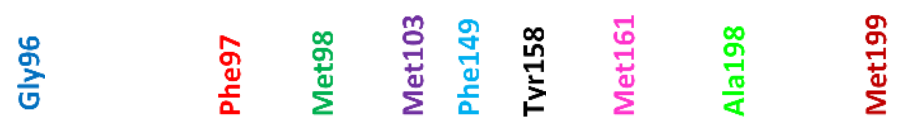

D

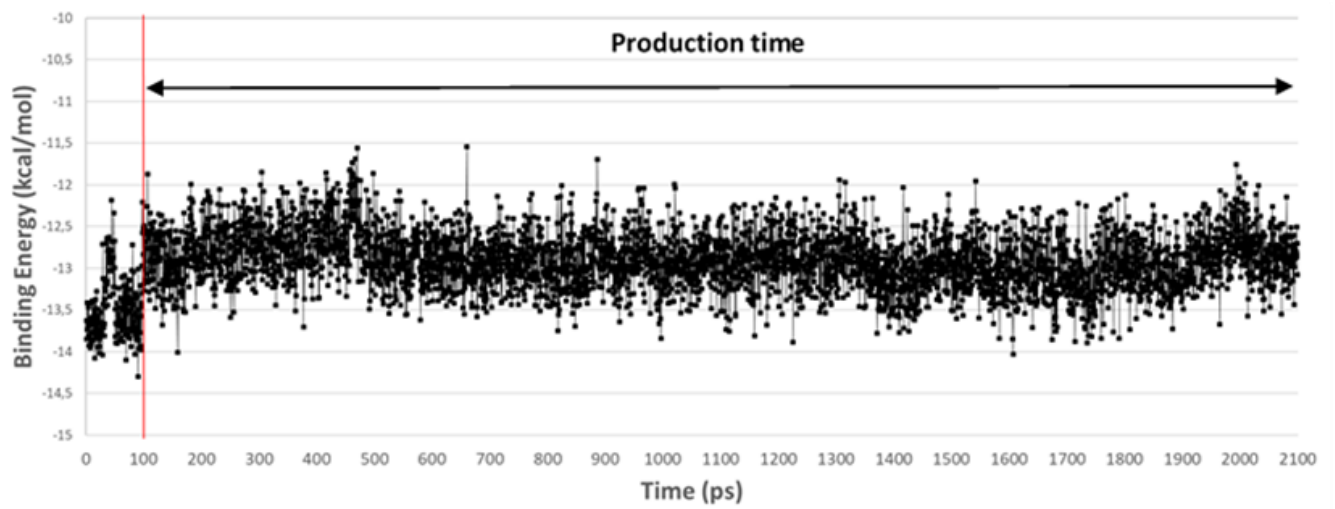

Figure 3. (A) The MtInhA (4TZK)-compound 7 (green) obtained from docking ( $t=0 \mathrm{~ns}$ ). NAD ${ }^{+}$is presented as a cofactor (pink). (B) The MtInhA (4TZK)-compound 7 after a 2 ns MD simulation. (C) The protein-ligand interaction fingerprint (PLIF) showing the interaction of reference ligand with the MtInhA (4TZK) binding pocket residues during the simulation as barcodes (a black line indicating the presence of the interaction at snapshot). (D) The binding energy $(\mathrm{kJ} / \mathrm{mol})$ between reference ligand and MtInhA (4TZK). "d" indicates backbone donor interactions, "a" indicates backbone acceptor interactions, "R" indicates H-arene interactions and " $\mathrm{D}$ " indicates side chain donor interactions. Two interactions of the same type per residue (for example " $\mathrm{R}$ " for Gly96) indicate that multiple interactions of the specified type between ligand and residue are formed. "*" indicates the strong interactions. The arrow indicates the sequence of snapshots of every $50 \mathrm{ps}$ from 0 to $2 \mathrm{~ns}$. 
A

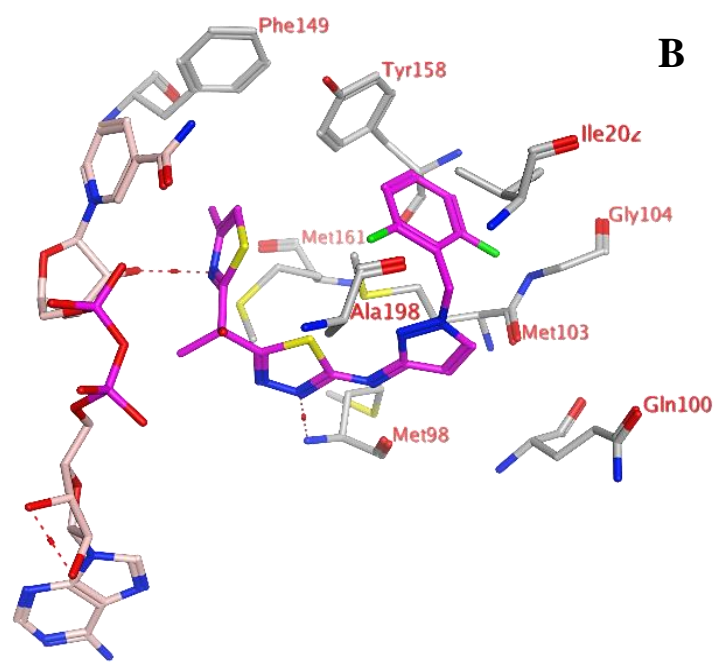

B

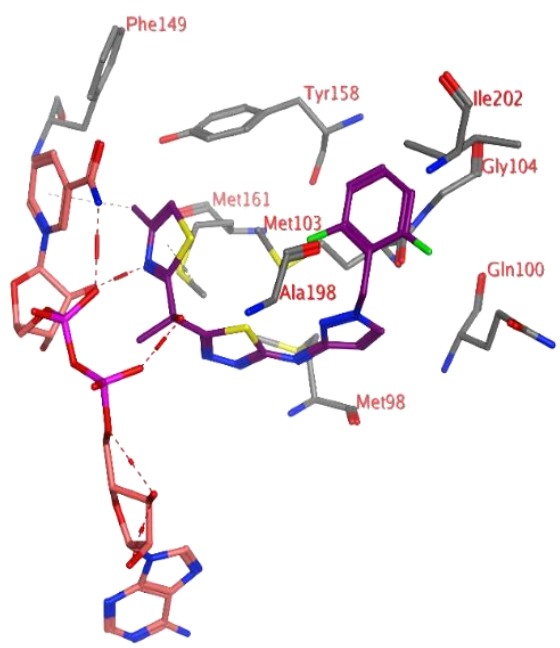

C

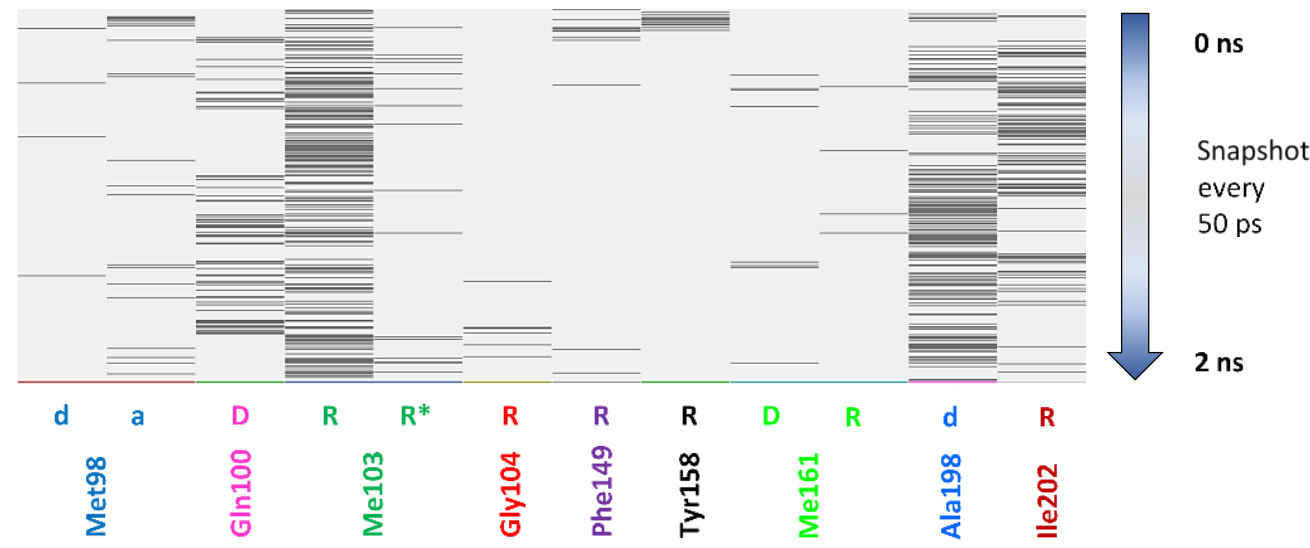

D

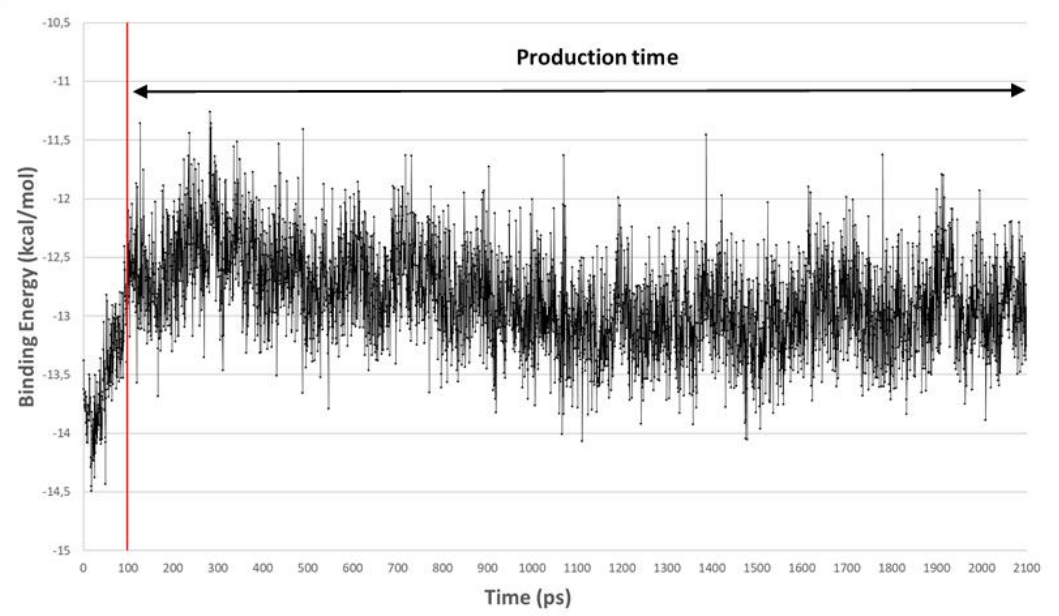

Figure 4. (A) The MtInhA (4BQP)-reference ligand (indicated with purple) $(t=0 \mathrm{~ns})$. $\mathrm{NAD}^{+}$is presented as a cofactor (pink). (B) The MtInhA (4BQP)-reference ligand after a 2 ns MD simulation. (C) The protein-ligand interaction fingerprint (PLIF) showing the interaction of reference ligand with the MtInhA (4BQP) binding pocket residues during the simulation as barcodes (a black line indicating the presence of the interaction at snapshot). (D) The binding energy ( $\mathrm{kJ} / \mathrm{mol}$ ) between reference ligand and MtInhA (4BQP). "d" indicates backbone donor interactions, "a" indicates backbone acceptor interactions, " $D$ " indicates side chain donor interactions, and " $\mathrm{R}$ " indicates $\mathrm{H}$-arene interactions. Two interactions of the same type per residue (for example " $R$ " for Met103) indicate that multiple interactions of the specified type between ligand and residue are formed. "*" indicates the strong interactions. The arrow indicates the sequence of snapshots of every $50 \mathrm{ps}$ from 0 to 2 ns. 

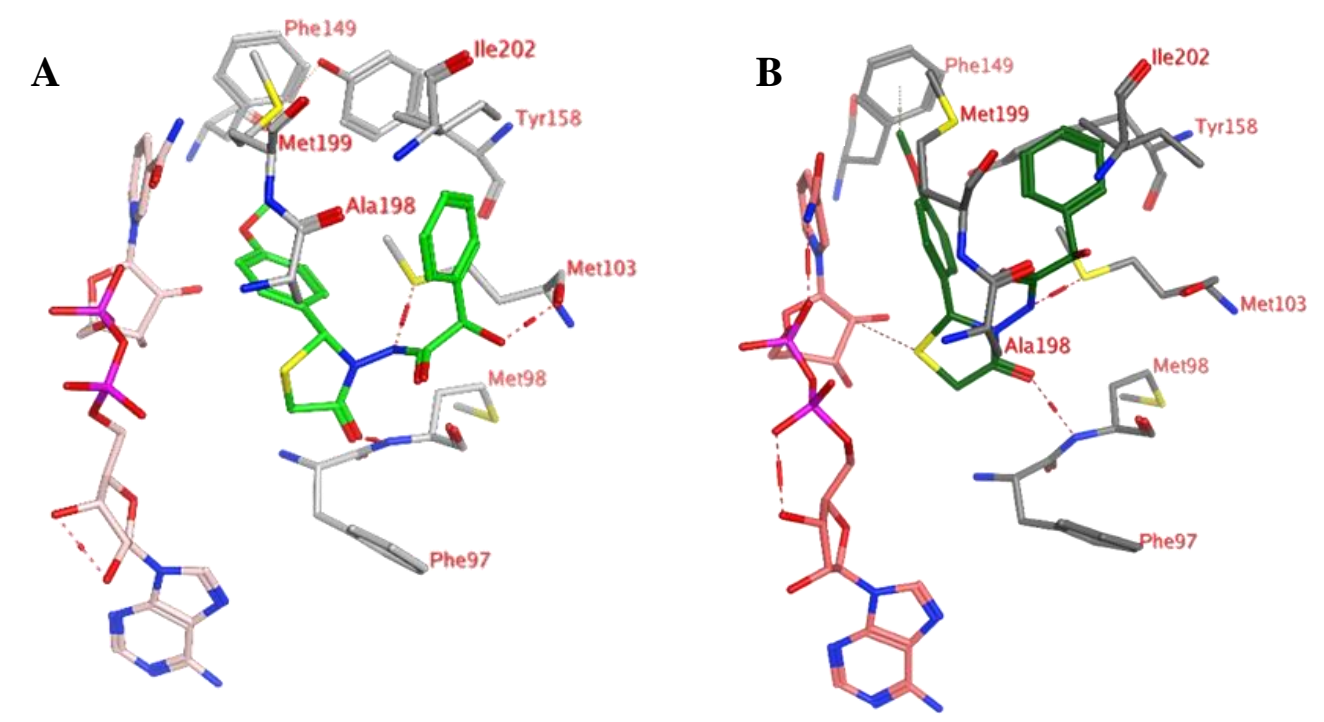

C

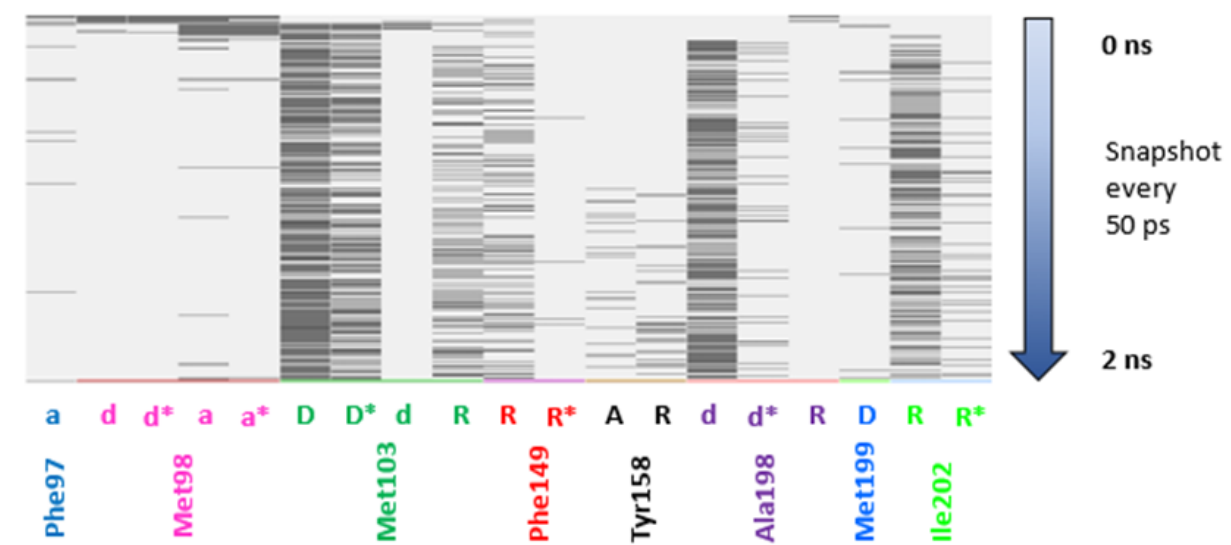

D

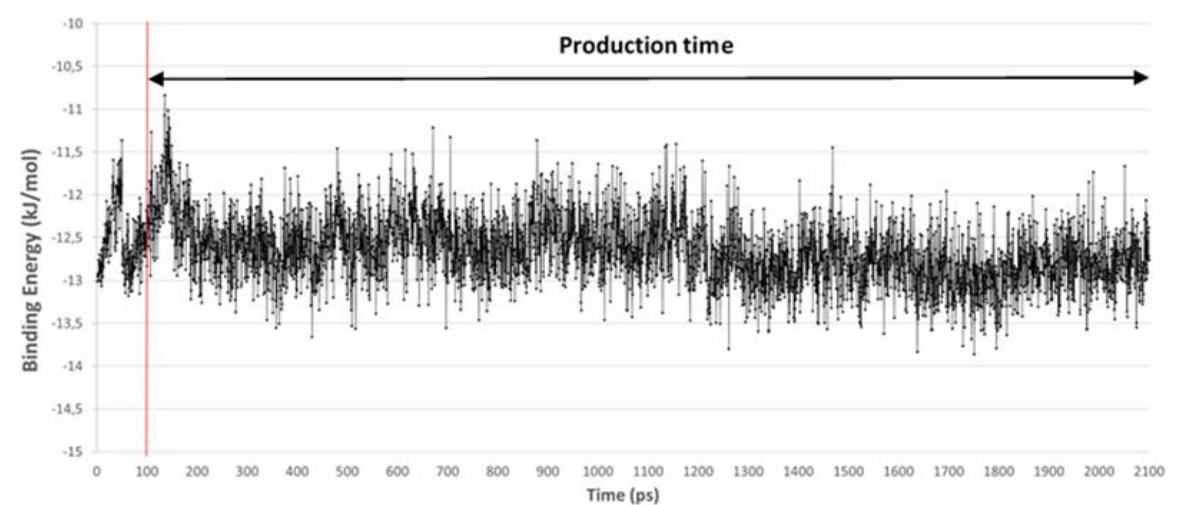

Figure 5. (A) The MtInhA (4BQP)-compound 7 (green) obtained from docking ( $t=0 \mathrm{~ns}$ ). $\mathrm{NAD}^{+}$is presented as a cofactor (pink). (B) The MtInhA (4BQP)-compound 7 after a 2 ns MD simulation. (C) The protein-ligand interaction fingerprint (PLIF) showing the interaction of reference ligand with the MtInhA (4BQP) binding pocket residues during the simulation as barcodes (a black line indicating the presence of the interaction at snapshot). (D). The binding energy ( $\mathrm{kJ} / \mathrm{mol}$ ) between ligand and MtInhA (4BQP). "a" indicates backbone acceptor interactions, " $\mathrm{d}$ " indicates backbone donor interactions, " $\mathrm{D}$ " indicates sidechain donor interactions, and " $\mathrm{R}$ " indicates $\mathrm{H}$-arene interactions, "A" indicates sidechain acceptor interactions. Two interactions of the same type per residue (for example " $\mathrm{D}$ " for Met103) indicate that multiple interactions of the specified type between ligand and residue are formed. "**" indicates the strong interactions. The arrow indicates the sequence of snapshots of every $50 \mathrm{ps}$ from 0 to $2 \mathrm{~ns}$. 
A hydrogen bond between thiadiazole nitrogen of the reference ligand and the hydroxyl group of $\mathrm{NAD}^{+}$observed in $96.4 \%$ of the production run snapshots. The hydroxyl group of the ligand may form two interactions but not the same time with oxygen atoms $\mathrm{O} 1 \mathrm{~N}$ and $\mathrm{O} 1 \mathrm{~A}$ of the phosphate groups at $\mathrm{NAD}^{+}$. The interactions were observed in $88.4 \%$ and $92.1 \%$ of all frames during $2 \mathrm{~ns}$ MD simulation, respectively (Figure 4B). It can be said that compound 7 may interact in general with $\mathrm{NAD}^{+}$in three ways. The calculated average binding energy between reference ligand and MtInhA (4BQP) was $-12.87 \mathrm{~kJ} / \mathrm{mol}$ (RMSD: $0.4323 \AA$ ) (Figure 4D).

Fourteen newly synthesized thiazolidinones (i.e., compounds 3-16) have been docked into the active site of the MtInhA (4BQP) crystal structure. Compound 7, as shown in Figure 5A, forms a strong a hydrogen bond through its hydroxyl group with the backbone of Met103. Another hydrogen bond between the sidechain of Met103 and the amide group of the ligand is observed. The carbonyl functionality of the thiazolidinone ring interacts with both Met198 and Phe97 by hydrogen bonds. $4-\mathrm{OCH}_{3}$ substituted phenyl may form hydrophobic interactions with sidechain of Phe149 and Tyr158, while 4$\mathrm{OCH}_{3}$ moiety may also form $\mathrm{H}$-arene interactions with only Phe149 side chain.

In MD simulation of compound 7 (represented as 40 snapshots; one snapshot per $50 \mathrm{ps}$ ), the detected main interactions between ligand and active site were Met103 ("D": sidechain donor interaction and "d": side chain acceptor interaction), Phe 149 ("R": H-Arene interaction), Ala198 ("D") and Ile202 ("R"). The H-arene interaction between the ligand and the sidechain of Ile202 was observed in $48 \%$ of the frames, including the first ( $0 \mathrm{~ns})$ and the last $(2 \mathrm{~ns})$. The hydrogen bond between the ligand and the sidechain of Met 103 was observed in $79 \%$ of the snapshots. Another formed hydrogen bond between the ligand and the backbone of Ala198 was observed in $63 \%$ of the frames, including the first $(0 \mathrm{~ns})$ and the last ( $2 \mathrm{~ns}$ ). In addition, an H-arene interaction formed by sidechain of Ile 202 is observed in $48 \%$ of the snapshots. Although not shown in Figure 5B, the interaction between the methoxy group of compound 7 and the hydroxyl group of $\mathrm{NAD}^{+}$is observed in $2.2 \%$ of the production run snapshots. As a result of the steric barrier of methyl, it may not possibly form a strong stable hydrogen bond between the ligand and $\mathrm{NAD}^{+}$(Figure 5B). The calculated average binding energy between compound 7 and MtInhA (4BQP) was $-12.6 \mathrm{~kJ} / \mathrm{mol}$ (RMSD: 0,3879 $\AA$ ) (Figure 5D).

As noted above, the docking studies suggest that the new compounds interact with the targeted enzymes MtInhA (4TZK) and (4BQP) like their reference ligands. According to the MD simulations, the binding energies of compound $\mathbf{7}$ is similar to the reference ligands. Therefore, it may be expected that compound 7 could show its antimycobacterial action through inhibiting both the targeted MtInhA enzymes.

\section{Conclusion}

In the present work, fourteen new 2-hydroxy- $N$-(4-oxo-2-substitutedphenyl-1,3-thiazolidin-3-yl)2-phenylacetamide derivatives were screened with in-vitro tests for their antimycobacterial activity against the pathogenic bacterium M. tuberculosis strain H37Rv. Compound $\mathbf{7}$ with a methoxy group at the para position of the phenyl ring exhibited the highest inhibition (98\%). In order to explain antimycobacterial action of the fourteen compounds, inhibition of the MtInhA enzymes (4TZK, 4BQP and 4BGE) were investigated with docking and dynamic simulations, as potential promising targets. For all MtInhA enzymes, all compounds were able to interact with the active sites in similar but not exactly the same ways with reference ligands. Compound 7, one of the top-ranked molecules in molecular modelling studies, showed strong interactions with the targeted enzymes and suggested as an inhibitor of MtInhA. Collectively, it is indicated that the inhibition of the MtInhA could be a part of the antimycobacterial activity of the new compounds and further development of these derivatives may present strong candidates for the treatment of tuberculosis.

\section{Acknowledgments}

Antimycobacterial data were provided by the Tuberculosis Antimicrobial Acquisition and Coordinating Facility (TAACF) through a research and development contract with the U.S. National Institute of Allergy and Infectious Diseases. Dr. Joseph A. Maddry (TAACF) and his team are recognized for their collaboration. This work was supported by the Research Fund of Istanbul University (Project number: 178/15012004). 


\section{Supporting Information}

Supporting information accompanies this paper on http://www.acgpubs.org/journal/organiccommunications.

\section{ORCID}

Özlen Güzel-Akdemir: 0000-0003-3680-1945

Kübra Demir-Yazıc1: 0000-0001-9928-4733

Muhammed Trawally: 0000-0002-0041-4612

Serap İpek Dingiş-Birgül: 0000-0003-2242-4296

Atilla Akdemir: $\underline{0000-0001-8416-0471}$

\section{References}

[1] Smith, I. Mycobacterium tuberculosis pathogenesis and molecular determinants of virulence. Clin Microbiol Rev. 2003, 16(3), 463-496.

[2] World Health Organization. Tuberculosis Homepage. available online: https://www.who.int/news$\mathrm{room} /$ fact-sheets/detail/tuberculosis (accessed on 5 May 2020)

[3] World Health Organization. Global tuberculosis reports. 2019, 23, 1-4.

[4] World Health Organization. Tuberculosis Fact Sheets. Available online: https://www.who.int/newsroom/fact-sheets/detail/tuberculosis (accessed on 5 May 2020).

[5] Glaziou, P. Predicted impact of the COVID-19 pandemic on global tuberculosis deaths in 2020. medRxiv. 2020, $5,1-7$.

[6] Zhang, Y.; Post-Martens, K.; Denkin, S. New drug candidates and therapeutic targets for tuberculosis therapy. Drug Discovery Today. 2006, 11, 21-27.

[7] Chan, E. D. Current medical treatment for tuberculosis. BMJ. 2002, 325(7375), 1282-1286.

[8] Gygli, S. M.; Borrell, S.; Trauner, A.; Gagneux, S. Antimicrobial resistance in Mycobacterium tuberculosis: mechanistic and evolutionary perspectives. FEMS Microbiol. Rev. 2017, 011, 354-373.

[9] Kanetaka, H.; Koseki, Y.; Taira, J.; Umei, T.; Komatsu, H.; Sakamoto, H.; Gulten, G.; Sacchettini, J. C.; Kitamura, M.; Aoki, S. Discovery of InhA inhibitors with anti-mycobacterial activity through a matched molecular pair approach. Eur. J. Med. Chem. 2015, 94, 378-385.

[10] Duan, X.; Xiang, X.; Xie, J. Crucial components of mycobacterium type II fatty acid biosynthesis (Fas-II) and their inhibitors. FEMS Microbiol. Lett. 2014, 360 (2), 87-99.

[11] Quémard, A.; Sacchettini, J. C.; Dessen, A.; Vilcheze, C.; Bittman, R.; Jacobs, W. R.; Blanchard, J. S. Enzymatic Characterization of the target for isoniazid in Mycobacterium tuberculosis. Biochemistry 1995, 34 (26), 8235-8241.

[12] Vigorita, M. G.; Ottanà, R.; Monforte, F.; Maccari, R.; Trovato, A.; Monforte, M. T.; Taviano, M. F. Synthesis and antiinflammatory, analgesic activity of 3,3'-(1,2-ethanediyl)-bis[2-aryl-4-thiazolidinone] chiral compounds. Bioorg. Med. Chem. Lett. 2001, 11 (21), 2791-2794.

[13] Vicini, P.; Geronikaki, A.; Anastasia, K.; Incerti, M.; Zani, F. Synthesis and antimicrobial activity of novel 2-thiazolylimino-5-arylidene-4-thiazolidinones. Bioorganic Med. Chem. 2006, 14 (11), 3859-3864.

[14] D’Ascenzio, M.; Bizzarri, B.; De Monte, C.; Carradori, S.; Bolasco, A.; Secci, D.; Rivanera, D.; Faulhaber, N.; Bordón, C.; Jones-Brando, L. Design, Synthesis and biological characterization of thiazolidin-4-one derivatives as promising inhibitors of Toxoplasma gondii. Eur. J. Med. Chem. 2014, 86, 17-30.

[15] Sonam; Chahal, V.; Kakkar, R. Theoretical study of the structural features and antioxidant potential of 4thiazolidinones. Struct. Chem. 2020, 1-10.

[16] Mishchenko, M.; Shtrygol, S.; Kaminskyy, D.; Lesyk, R. Thiazole-bearing 4-thiazolidinones as new anticonvulsant agents. Sci. Pharm. 2020, 88 (1), 16.

[17] Suryawanshi, R.; Jadhav, S.; Makwana, N.; Desai, D.; Chaturbhuj, D.; Sonawani, A.; Idicula-Thomas, S.; Murugesan, V.; Katti, S. B.; Tripathy, S.; et al. Evaluation of 4-thiazolidinone derivatives as potential reverse transcriptase inhibitors against HIV-1 drug resistant strains. Bioorg. Chem. 2017, 71, 211-218.

[18] De Monte, C.; Carradori, S.; Bizzarri, B.; Bolasco, A.; Caprara, F.; Mollica, A.; Rivanera, D.; Mari, E.; Zicari, A.; Akdemir, A.; et al. Anti-candida activity and cytotoxicity of a large library of new N-substituted1,3-Tthiazolidin-4-one derivatives. Eur. J. Med. Chem. 2016, 107, 82-96. 
[19] Türe, A.; Ergül, M.; Ergül, M.; Altun, A.; Küçükgüzel, İ. Design, synthesis, and anticancer activity of novel 4-thiazolidinone-phenylaminopyrimidine hybrids. Mol. Divers. 2020, 1, 3.

[20] Küçükgüzel, Ş. G.; Oruç, E. E.; Rollas, S.; Şahin, F.; Özbek, A. Synthesis, characterisation and biological activity of novel 4-thiazolidinones, 1,3,4-oxadiazoles and some related compounds. Eur. J. Med. Chem. 2002, 37 (3), 197-206.

[21] Babaoglu, K.; Page, M. A.; Jones, V. C.; McNeil, M. R.; Dong, C.; Naismith, J. H.; Lee, R. E. Novel inhibitors of an emerging target in Mycobacterium tuberculosis;substituted thiazolidinones as inhibitors of DTDP-rhamnose synthesis. Bioorganic Med. Chem. Lett. 2003, 13 (19), 3227-3230.

[22] Güzel-Akdemir, Ö.; Angeli, A.; Demir, K.; Supuran, C. T.; Akdemir, A. Novel thiazolidinone-containing compounds, without the well-known sulphonamide zinc-binding group acting as human carbonic anhydrase IX Iinhibitors. J. Enzyme Inhib. Med. Chem. 2018, 33 (1), 1299-1308.

[23] Güzel-Akdemir, Ö.; Carradori, S.; Grande, R.; Demir-Yazıc1, K.; Angeli, A.; Supuran, C. T.; Akdemir, A. Development of thiazolidinones as fungal carbonic anhydrase inhibitors. Int. J. Mol. Sci. 2020, 21 (8), 2960.

[24] Zieliński, W.; Kudelko, A.; Czardybon, W. The synthesis of 4-acylamino-1,2,4-triazole derivatives in the reaction of $\alpha$-hydroxyacid hydrazides and orthoesters. J. Heterocycl. Chem. 2005, 42 (7), 1393-1397.

[25] Inderlied, C. B. Antimycobacterial susceptibility testing: present practices and future trends. Eur. J. Clin. Microbiol. Infect. Dis. 1994, 980-993.

[26] Demir-Yazıcı, K.; Güzel-Akdemir, Ö.; Angeli, A.; Supuran, C. T.; Akdemir, A. Novel indole-based hydrazones as potent inhibitors of the $\alpha$-class carbonic anhydrase from pathogenic bacterium Vibrio cholerae. Int. J. Mol. Sci. 2020, 21 (9), 3131.

[27] Halgren, T. A. Merck molecular force field. I. basis, form, scope, parameterization, and performance of MMFF94. J. Comput. Chem. 1996, 17 (5-6), 490-519.

[28] Labute, P. Protonate3D: Assignment of ionization states and hydrogen coordinates to macromolecular structures. Proteins Struct. Funct. Bioinforma. 2009, 75 (1), 187-205.

[29] Case, D.A.; Babin, V.; Berryman, J.T., Betz, R.M.; Cai, Q.; Cerutti, D.S.; Cheatham, T.E. III. Darden, T.A.; Duke, R.E.; Gohlke, H.; Goetz, A.W.; Gusarov, S.; Homeyer, N.; Janowski, P.; Kaus, J.; Kolossváry, I.; Kovalenko, A.; Lee, T.S.; LeGrand, S.; Luchko, T.; Luo, R.; Madej, B.; Merz, K.M.; Paesani, F.; Roe, D.R.; Roitberg, A.; Sagui, C.; Salomon-Ferrer, R.; Seabra, G.; Simmerling, C.L.; Smith, W.; Swails, J.; Walker, R.C.; Wang, J.; Wolf, R.M.; Wu, X.; Kollman P. AMBER 14, (University of California, San Francisco) 2014, $1-814$.

[30] Labute, P. The Generalized born/volume integral implicit solvent model: estimation of the free energy of hydration using London dispersion instead of atomic surface area. J. Comput. Chem. 2008, 29 (10), 16931698.

[31] Phillips, J. C.; Braun, R.; Wang, W.; Gumbart, J.; Tajkhorshid, E.; Villa, E.; Chipot, C.; Skeel, R. D.; Kalé, L.; Schulten, K. Scalable molecular dynamics with NAMD. J. Comput. Chem. 2005, 33(1), 1781-1802.

[32] Taylor, P. J. The infra-red spectroscopy of some 2-substituted thiazolid-4-ones, a new class of enaminoketone-II. infra-red assignments and chemical implications. Spectrochim. Acta Part A Mol. Spectrosc. 1970, 26 (1), 165-194.

[33] Kaynak, F. B.; Öztürk, D.; Özbey, S.; Çapan, G. New N'-alkylidene/cycloalkylidene derivatives of 5methyl-3-phenyl- 1H-indole-2-carbohydrazide: synthesis, crystal structure, and quantum mechanical calculations. J. Mol. Struct. 2005, 740 (1-3), 213-221.

[34] Güzel, Ö.; Ilhan, E.; Salman, A. Synthesis and antimycobacterial activity of new 2-hydroxy-N-(3-oxo-1thia-4-azaspiro[4.4]non-4-yl)/(3-oxo-1-thia-4-azaspiro[4.5]dec-4-Yl)-2, 2-diphenylacetamide derivatives. Monatsh Chem. 2006, 137 (6), 795-801.

[35] Verma, A.; Saraf, S. K. 4-Thiazolidinone - a biologically active scaffold. Eur. J. Med. Chem. 2008, 43(5), 897-905.

[36] Gupta, A.; Singh, R.; Sonar, P. K.; Saraf, S. K. Novel 4-thiazolidinone derivatives as anti-infective agents: synthesis, characterization, and antimicrobial evaluation. Biochem. Res. Int. 2016, 2016, 1-8.

[37] Ergenç, N.; Çapan, G.; Günay, N. S.; Özkirimli, S.; Güngör, M.; Özbey, S.; Kendi, E. Synthesis and hypnotic activity of new 4-thiazolidinone and 2-thioxo- 4,5-imidazolidinedione derivatives. Arch. Pharm. (Weinheim). 1999, 332 (10), 343-347.

[38] Karali, N.; Ilhan, E.; Gürsoy, A.; Kiraz, M. New cyclohexylidenehydrazide and 4-aza-1-thiaspiro[4.5] decan-3-one derivatives of 3-phenyl-4 (3H)-quinazolinones. Farmaco 1998, 53 (5), 346-349.

[39] He, X.; Alian, A.; Stroud, R.; Ortiz De Montellano, P. R. Pyrrolidine carboxamides as a novel Class of inhibitors of enoyl acyl carrier protein reductase from Mycobacterium tuberculosis. J. Med. Chem. 2006, 49 (21), 6308-6323.

[40] Vilchèze, C.; Wang, F.; Arai, M.; Hazbón, M. H.; Colangeli, R.; Kremer, L.; Weisbrod, T. R.; Alland, D.; Sacchettini, J. C.; Jacobs, W. R. Transfer of a point mutation in Mycobacterium tuberculosis InhA resolves the target of isoniazid. Nat. Med. 2006, 12 (9), 1027-1029. 
[41] Dessen, A.; Quémard, A.; Blanchard, J. S.; Jacobs, W. R.; Sacchettini, J. C. Crystal structure and function of the isoniazid target of Mycobacterium tuberculosis. Science (80-. ). 1995, 267 (5204), 1638-1641.

[42] Jeffrey, George A.; An introduction to hydrogen bonding, Oxford University Press, New York, 1997.

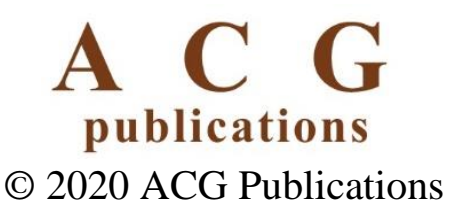

